\title{
COLLAPSING OF CALABI-YAU MANIFOLDS AND SPECIAL LAGRANGIAN SUBMANIFOLDS
}

\author{
BY Yuguang Zhang
}

\begin{abstract}
In this paper, the relationship between the existence of special Lagrangian submanifolds and the collapsing of Calabi-Yau manifolds is studied. First, special Lagrangian fibrations are constructed on some regions of bounded curvature and sufficiently collapsed in Ricci-flat CalabiYau manifolds. Then, conversely, it is shown that the existence of special Lagrangian submanifolds with small volume implies the collapsing of some regions in the ambient Calabi-Yau manifolds.
\end{abstract}

1. Introduction. The notion of the special Lagrangian submanifold was introduced by Harvey and Lawson in the seminal paper [26]. Mclean studied the deformation theory of special Lagrangian submanifolds in [33. In the pioneer work [41], Stominger, Yau and Zaslow propose a conjecture about constructing the mirror manifold of a given Calabi-Yau manifold, the SYZ conjecture, via special Lagrangian fibrations. Since then, lots of works have been devoted to studying special Lagrangian submanifolds and fibrations (cf. $18-20,27,29,30,32,36-38,40,42$, and references in $[30])$. In $[31]$ and $[23]$, a refined version of SYZ conjecture was proposed by using the collapsing of Ricci-flat Calabi-Yau manifolds in the Gromov-Hausdorff sense. These two versions of SYZ conjecture suggest a relationship between the existence of special Lagrangian submanifolds and the collapsing of Calabi-Yau manifolds. In this paper, we study this relationship.

If $(M, \omega, J, g)$ is a compact Ricci-flat Kähler $n$-manifold, and admits a nowhere vanishing holomorphic $n$-form $\Omega$ (the holomorphic volume form), $(M, \omega, J, g, \Omega)$ is called a Ricci-flat Calabi-Yau $n$-manifold, and $(\omega, J, g, \Omega)$ is 
called a Calabi-Yau structure on $M$. We can normalize $\Omega$ in the following way

$$
\frac{\omega^{n}}{n !}=\frac{(-1)^{\frac{n^{2}}{2}}}{2^{n}} \Omega \wedge \bar{\Omega}
$$

(cf. [30]). Yau's theorem on Calabi conjecture guarantees the existence of Ricci-flat Kähler metrics on Kähler manifolds with trivial canonical bundle (cf. [44]), which implies the existence of Calabi-Yau structures on such manifolds. The holonomy group of a Ricci-flat Calabi-Yau $n$-manifold is a subgroup of $S U(n)$. The study of Calabi-Yau manifolds is important in both mathematics and physics (cf. 45]).

A special Lagrangian submanifold $L$ of phase $\theta \in \mathbb{R}$ in a Ricci-flat CalabiYau $n$-manifold $(M, \omega, J, g, \Omega)$ is a Lagrangian submanifold $L \subset M$ corresponding to the Kähler form $\omega$ so that $\left.\operatorname{Re} e^{\sqrt{-1} \theta} \Omega\right|_{L}=d v_{\left.g\right|_{L}}$ where $d v_{g_{L}}$ denotes the volume form of $\left.g\right|_{L}$ on $L$. Equivalently, $\operatorname{dim}_{\mathbb{R}} L=n$,

$$
\left.\omega\right|_{L} \equiv 0,\left.\quad \operatorname{Im} e^{\sqrt{-1} \theta} \Omega\right|_{L} \equiv 0
$$

(cf. [26]). In [33], Mclean showed that, for a compact special Lagrangian submanifold $L$ in a Calabi-Yau manifold $(M, \omega, J, g, \Omega)$, the local moduli space of special Lagrangian submanifolds near $L$ is a smooth manifold of dimension $b_{1}(L)$, and, moreover, the tangent space of the moduli space at $L$ can be identified with the space of harmonic 1-forms on $\left(L,\left.g\right|_{L}\right)$. In [27, various structures on the moduli space of special Lagrangian submanifolds were studied.

A special Lagrangian fibration on a Calabi-Yau $n$-manifold $(M, \omega, \Omega)$ consists of a topological space $B$ and a surjection $f: M \longrightarrow B$ such that there is an open dense subset $B_{0} \subset B$, which is a real $n$-manifold, such that, for any $b \in B_{0}, f^{-1}(b)$ is a smooth special Lagrangian submanifold in $(M, \omega, \Omega)$. By 13] (see also [21]), $f^{-1}(b), b \in B_{0}$, is an $n$-torus. The SYZ conjecture asserts the existence of special Lagrangian fibrations on a Calabi-Yau manifold whose complex structure is close enough to the large complex structure limit point (cf. 41]). The mirror manifold is a compactification of the dual fibration of $f: f^{-1}\left(B_{0}\right) \longrightarrow B_{0}$. Generalized special Lagrangian fibrations were constructed in some almost Calabi-Yau manifolds in $[\mathbf{1 9}, 36-38$. In $[\mathbf{2 2}]$, special Lagrangian fibrations were constructed on some Borcea-Voisin type Calabi-Yau 3-manifolds with degenerated Ricci-flat Kähler-Einstein metrics.

In 31] and 23, SYZ conjecture was refined to the following form: Let $\pi: \mathcal{M} \rightarrow \Delta$ be a maximally unipotent degeneration of Calabi-Yau $n$-manifolds over the unit disc $\Delta \subset \mathbb{C}$, and $\alpha$ be an ample class on $\mathcal{M}$. For any $t \in \Delta \backslash\{0\}$, let $\tilde{g}_{t}$ be the unique Ricci-flat Kähler metric on $M_{t}=\pi^{-1}(t)$ with its Kähler form $\left.\tilde{\omega}_{t} \in \alpha\right|_{M_{t}} \in H^{1,1}\left(M_{t}, \mathbb{R}\right) \cap H^{2}\left(M_{t}, \mathbb{Z}\right)$, and $\bar{g}_{t}=\operatorname{diam}_{\tilde{g}_{t}}^{-2}(M) \tilde{g}_{t}$. Then $\left(M_{t}, \bar{g}_{t}\right)$ converges to a compact metric space $\left(B, d_{B}\right)$ of Hausdorff dimension $n$ in the Gromov-Hausdorff sense, when $t \rightarrow 0$. Furthermore, there is a closed 
subset $S_{B} \subset B$ of Hausdorff dimension $n-2$ such that $B \backslash S_{B}$ is an affine manifold, and $d_{B}$ is induced by a Monge-Ampère metric $g_{B}$ on $B \backslash S_{B}$ (cf. [31]). The mirror manifolds are supposed to be constructed from the dual affine structure on $B \backslash S_{B}$ and the metric $g_{B}$. This conjecture was verified for some K3 surfaces in 23 . By using hyper-Kähler rotation, some K3 surfaces admit special Lagrangian fibrations. It was shown in 23 that some K3 surfaces with Ricci-flat Kähler metrics collapse along such special Lagrangian fibrations. The two versions of SYZ conjecture suggest the equivalence between the existence of special Lagrangian submanifolds and the collapsing of Ricci-flat Kähler metrics on some regions of Calabi-Yau manifolds, when complex structures are close enough to the large complex limit point.

In Riemannian geometry, the collapsing of Riemannian manifolds has been studied by various authors (cf. $[\mathbf{8}-\mathbf{1 0}, \mathbf{1 2},[\mathbf{1 4}]$, and references in $[\mathbf{1 4}]$ ), since Gromov introduced the notion of Gromov-Hausdorff topology in [17]. In [10], it was proved that there is a constant $\epsilon_{0}(n)>0$ depending only on $n$ such that there is an $F$-structure of positive rank on the region $M_{\epsilon_{0}}$ in a Riemannian $n$-manifold $(M, g)$, where $M_{\epsilon_{0}}$ denotes the subset with injectivity radius $i_{g}(p)<\epsilon_{0}$ and sectional curvature $\sup _{B_{g}(p, 1)}\left|K_{g}\right| \leq 1$, for any $p \in M_{\epsilon_{0}}$. See $\overline{9}$ and [10] for the definition of an $F$-structure of positive rank, which is a generalization of fibration. A folklore conjecture says that there should be special Lagrangian fibrations on such region in a Calabi-Yau manifold, i.e. the region of bounded curvature and sufficiently collapsed (cf. [15]). The first result in the present paper is devoted to constructing special Lagrangian fibrations under such Riemannian geometric conditions.

Theorem 1.1. For any $n \in \mathbb{N}$ and any $\sigma>1$, there exists a constant $\epsilon=\epsilon(n, \sigma)>0$ depending only on $n$ and $\sigma$ such that, if $(M, \omega, J, g, \Omega)$ is a closed Ricci-flat Calabi-Yau n-manifold with $[\omega] \in H^{2}(M, \mathbb{Z})$, and $p \in M$ such that

i) the injectivity radius and the sectional curvature

$$
i_{g}(p)<\epsilon, \quad \sup _{B_{g}(p, 1)}\left|K_{g}\right| \leq 1,
$$

ii) $\left[\left.\Omega\right|_{B_{g}\left(p, \sigma i_{g}(p)\right)}\right] \neq 0$ in $H^{n}\left(B_{g}\left(p, \sigma i_{g}(p)\right), \mathbb{C}\right)$,

then there is an open subset $W \subset M$ satisfying $B_{g}\left(p, \sigma i_{g}(p)\right) \subset W$, and $(W, \omega, \Omega)$ admits a special Lagrangian fibration of a phase $\theta \in \mathbb{R}$, i.e. there is a topological space $B$, and a surjection $f: W \longrightarrow B$ such that, for any $b \in B, f^{-1}(b)$ is a smooth $n$-submanifold,

$$
\left.\omega\right|_{f^{-1}(b)} \equiv 0, \quad \text { and }\left.\operatorname{Im} e^{\sqrt{-1} \theta} \Omega\right|_{f^{-1}(b)} \equiv 0 .
$$


Remark 1.2. From the proof of this theorem, we can see that $B$ is an orbifold, and, if $b$ belongs to the singular set of $B, f^{-1}(b)$ is a smooth multifiber.

REMARK 1.3. Condition ii) in the theorem can be replaced by the following small non-vanishing $n$-cycle condition: there is an $[A] \in H_{n}\left(B_{g}\left(p, \sigma i_{g}(p)\right), \mathbb{Z}\right)$ such that

$$
\int_{A} \Omega \neq 0
$$

This condition cannot be removed since it is satisfied if there is a special Lagrangian submanifold $L$ near $p$ having size comparable with $i_{g}(p)$, for example $L \subset B_{g}\left(p, \sigma i_{g}(p)\right)$.

REMARK 1.4. It is a challenging task to verify condition i) in Theorem 1.1. i.e. to find the region of bounded curvature in a Ricci-flat Calabi-Yau manifold. It was shown in $\mathbf{1 2}$ that if $(M, \omega, J, g, \Omega)$ is a K3-surface with Ricci-flat metric, there are universal constants $C>0, \tau>0$, and a finite subset $\left\{p_{j}\right\} \subset M, 1 \leq j \leq \tau$, such that

$$
\sup _{B_{g}(p, 1)}\left|K_{g}\right| \leq C,
$$

for any $p \in M \backslash \bigcup_{1 \leq j \leq \tau} B_{g}\left(p_{j}, 2\right)$.

Next, in the opposite direction, we show that the existence of special Lagrangian submanifolds with small volume implies the collapsing of some regions in the ambient Calabi-Yau manifolds. The following theorem is a corollary of the volume comparison theorem for calibrated submanifolds in [19].

Theorem 1.5. Let $(M, \omega, J, g, \Omega)$ be a closed Ricci-flat Calabi-Yau n-manifold, and $p \in M$. Assume that the sectional curvature $K_{g}$ satisfies

$$
\sup _{B_{g}(p, 2 \pi)} K_{g} \leq 1
$$

and there is a special Lagrangian submanifold $L$ of phase $\theta$ such that $p \in L$, and

$$
\int_{L} \operatorname{Re} e^{\sqrt{-1} \theta} \Omega<\frac{\pi}{2 n} \varpi_{n-1}
$$

where $\varpi_{n-1}$ denotes the volume of $S^{n-1}$ with the standard metric of constant curvature 1 . Then the injectivity radius $i_{g}(p)$ of $(M, g)$ at $p$ satisfies

$$
i_{g}(p)^{n} \leq \frac{n \pi^{n-1}}{2^{n-1} \varpi_{n-1}} \int_{L} \operatorname{Re} e^{\sqrt{-1} \theta} \Omega
$$


As mentioned in Remark 1.4, the assumption of bounded curvature is not necessary. If we strengthen the condition of the existence of a special Lagrangian submanifold to the existence of a special Lagrangian fibration, we can still obtain the collapsing result in the absence of bounded curvature.

THEOREM 1.6. For any $n \in \mathbb{N}$ and any $\varepsilon>0$, there is a constant $\delta=$ $\delta(n, \varepsilon)>0$ such that the following statement is true: Assume that $(M, \omega, J, g, \Omega)$ is a closed Ricci-flat Calabi-Yau n-manifold, $p \in M$, and there is a homology class $A \in H_{n}(M, \mathbb{Z})$ such that, for any $x \in B_{g}(p, 1)$, there is a special Lagrangian submanifold $L_{x}$ of phase $\theta$ passing $x$ and presenting $A$, i.e. $x \in L_{x}$ and $\left[L_{x}\right]=A$. If

then

$$
\int_{A} \operatorname{Re} e^{\sqrt{-1} \theta} \Omega<\delta
$$

$$
\operatorname{Vol}_{g}\left(B_{g}(p, 1)\right) \leq \varepsilon .
$$

Theorem 1.1. Theorem 1.5 and Theorem 1.6 give an evidence of the equivalence between the existence of special Lagrangian submanifolds and the collapsing of Ricci-flat Kähler metrics on Calabi-Yau manifolds near the large complex limit point from the Riemannian geometry's point of view.

The organization of the paper is as follows: In $\S 2$, we review some notions and results, which will be used in this paper. In $\S 3$, we use the blow-up argument to give local approximations of Calabi-Yau manifolds by complete flat Calabi-Yau manifolds. In $\S 4$, we study the deformation of special Lagrangian fibrations. In $\S 5$, we prove Theorem 1.1 by combining the results in $\S 3$ and $\S 4$. Finally, we prove Theorem 1.5 and Theorem 1.6 in $\S 6$.

Acknowledgement: The author would like to thank Prof. Weidong Ruan and Prof. Xiaochun Rong for useful discussions. Thanks also goes to Prof. Fuquan Fang for his continuous support.

2. Preliminaries. In this section, we review some notions and results which will be used in the proof of Theorem 1.1, Theorem 1.5 and Theorem 1.6.

2.1. Cheeger-Gromov convergence. Since Gromov introduced the concept of Gromov-Hausdorff convergence in [17, the convergence of Riemannian manifolds has been studied from various perspectives (cf. $[\mathbf{1}, \mathbf{2}, \mathbf{1 1}, \mathbf{1 2}, \mathbf{1 4}, \mathbf{1 6}, \mathbf{2 3}$, 39, 43 and references in [4]). There is an extension of Gromov-Hausdorff convergence to sequences of pointed metric spaces for dealing with non-compact situations.

Definition $2.1(\mathbf{1 7}, \mathbf{1 4})$. For two pointed complete metric spaces $\left(X, d_{X}, x\right)$ and $\left(Y, d_{Y}, y\right)$, a map $\psi:(X, x) \rightarrow(Y, y)$ is called an $\epsilon$-pointed approximation if $\psi\left(B_{d_{X}}\left(x, \epsilon^{-1}\right)\right) \subset B_{d_{Y}}\left(y, \epsilon^{-1}\right), \psi(x)=y$, and $\left.\psi\right|_{B_{d_{X}}\left(x, \epsilon^{-1}\right)}$ : $B_{d_{X}}\left(x, \epsilon^{-1}\right) \longrightarrow B_{d_{Y}}\left(y, \epsilon^{-1}\right)$ is an $\epsilon$-approximation, i.e.

$$
B_{d_{Y}}\left(y, \epsilon^{-1}\right) \subset\left\{y^{\prime} \in Y \mid d_{Y}\left(y^{\prime}, \psi\left(B_{d_{X}}\left(x, \epsilon^{-1}\right)\right)\right)<\epsilon\right\},
$$


and

$$
\left|d_{X}\left(x_{1}, x_{2}\right)-d_{Y}\left(\psi\left(x_{1}\right), \psi\left(x_{2}\right)\right)\right|<\epsilon
$$

for any $x_{1}$ and $x_{2} \in B_{d_{X}}\left(x, \epsilon^{-1}\right)$. The number

$d_{G H}\left(\left(X, d_{X}, x\right),\left(Y, d_{Y}, y\right)\right)=\inf \left\{\begin{array}{l|l}\epsilon \mid \begin{array}{c}\text { There are } \epsilon \text {-pointed approximations } \\ \psi:(X, x) \rightarrow(Y, y), \text { and } \phi:(Y, y) \rightarrow(X, x)\end{array}\end{array}\right\}$ is called pointed Gromov-Hausdorff distance between $\left(X, d_{X}, x\right)$ and $\left(Y, d_{Y}, y\right)$. We say that a family of pointed complete metric spaces $\left(X_{k}, d_{X_{k}}, x_{k}\right)$ converges to a complete metric space $\left(Y, d_{Y}, y\right)$ in the pointed Gromov-Hausdorff sense, if

$$
\lim _{k \rightarrow \infty} d_{G H}\left(\left(X_{k}, d_{X_{k}}, x_{k}\right),\left(Y, d_{Y}, y\right)\right)=0 .
$$

The following is the famous Gromov pre-compactness theorem:

TheOREM $2.2(\mathbf{1 7}])$. Let $\left\{\left(M_{k}, g_{k}, p_{k}\right)\right\}$ be a family of pointed complete Riemannian manifolds such that Ricci curvatures $\operatorname{Ric}\left(g_{k}\right) \geq-C$ for a constant $C$ independent of $k$. Then, a subsequence of $\left(M_{k}, g_{k}, p_{k}\right)$ converges to a pointed complete path metric space $\left(Y, d_{Y}, y\right)$ in the pointed Gromov-Hausdorff sense.

The structure of the limit space was studied in $[\mathbf{5}, \mathbf{7}, \mathbf{1 1}, \mathbf{1 2}]$ et al. We need the following result in the proof of Theorem 1.6 .

Theorem $2.3([\mathbf{5}],[\mathbf{4}])$. Let $\left\{\left(M_{k}, g_{k}, p_{k}\right)\right\}$ be a family of pointed complete Ricci-flat Einstein n-manifolds, i.e. $\operatorname{Ric}\left(g_{k}\right) \equiv 0$, such that

$$
\operatorname{Vol}_{g_{k}}\left(B_{g_{k}}\left(p_{k}, 1\right)\right) \geq C,
$$

for a constant $C$ independent of $k$, and $\left(Y, d_{Y}, y\right)$ be a pointed complete path metric space such that

$$
\lim _{k \rightarrow \infty} d_{G H}\left(\left(M_{k}, g_{k}, p_{k}\right),\left(Y, d_{Y}, y\right)\right)=0 .
$$

Then the Hausdorff dimension $\operatorname{dim}_{\mathcal{H}} Y=n$, and there is a closed subset $S_{Y} \subset$ $Y$ of Hausdorff dimension $\operatorname{dim}_{\mathcal{H}} S_{Y}<n-1$ such that $Y \backslash S_{Y}$ is an n-manifold, and $d_{Y}$ is induced by a Ricci-flat Einstein metric $g_{\infty}$ on $Y \backslash S_{Y}$. Furthermore, for any compact subset $D \subset Y \backslash S_{Y}$, there are embeddings $F_{k, D}: D \longrightarrow M_{k}$ such that $F_{k, D}^{*} g_{k}$ converges to $g_{\infty}$ in the $C^{\infty}$-sense.

In $[\mathbf{1 7}$ and $[\mathbf{1 6}$, a convergence theorem, the Cheeger-Gromov convergence theorem, was proved for non-collapsed Riemannian manifolds with bounded curvature. The Kähler version of this theorem can be found in [35]. See [11 for the convergence of manifolds with other holonomy groups.

Theorem 2.4 (Kähler version of Cheeger-Gromov convergence theorem). Let $\left\{\left(M_{k}, g_{k}, J_{k}, \omega_{k}, p_{k}\right)\right\}$ be a family of pointed compact Kähler n-manifolds with sectional curvature and injectivity radius at $p_{k}$

$$
\left|K_{g_{k}}\right| \leq 1, \quad i_{g_{k}}\left(p_{k}\right) \geq C,
$$


for a constant $C>0$ independent of $k$. Then a subsequence $\left\{\left(M_{k}, g_{k}, J_{k}, \omega_{k}, p_{k}\right)\right\}$ converges to a complete Kähler n-manifold $(X, g, J, \omega, p)$ in the pointed $C^{1, \alpha_{-}}$ sense, i.e. for any $r>0$, there are embeddings $F_{k, r}: B_{g}(p, r) \longrightarrow M_{k}$ such that $F_{k, r}(p)=p_{k}, F_{k, r}^{*} g_{k}$ (resp. $d F_{k, r}^{-1} J_{k} d F_{k, r}$ and $F_{k, r}^{*} \omega_{k}$ ) converges to $g$ (resp. $J$ and $\omega)$ in the $C^{1, \alpha}$-sense.

In [1] it is shown that if we assume $g_{k}$ to be Einstein metrics then, by passing to a subsequence, $\left\{\left(M_{k}, g_{k}, J_{k}, \omega_{k}, p_{k}\right)\right\}$ converges to $(X, g, J, \omega, p)$ in the pointed $C^{\infty}$-sense, and $g$ is an Einstein metric too, i.e. $F_{k, r}^{*} g_{k}$ (resp. $d F_{k, r}^{-1} J_{k} d F_{k, r}$ and $F_{k, r}^{*} \omega_{k}$ ) converges to $g$ (resp. $J$ and $\omega$ ) in the $C^{\infty}$-sense. Assume that $\left(M_{k}, g_{k}, J_{k}, \omega_{k}, p_{k}\right)$ are Ricci-flat Calabi-Yau manifolds and $\Omega_{k}$ are the corresponding holomorphic volume forms. Since $\Omega_{k}$ are parallel, i.e. $\nabla^{g_{k}} \Omega_{k} \equiv 0$, for any $r>0, F_{k, r}^{*} \Omega_{k}$ converge to a holomorphic volume form $\Omega$ on $X$ in the $C^{\infty}$-sense, and $(X, g, J, \omega, \Omega)$ is a complete Ricci-flat Calabi-Yau $n$-manifold.

In $[\mathbf{9}$, [10, the collapsing of Riemannian manifolds with bounded curvature was studied by combining blow-up arguments and the Cheeger-Gromov convergence theorem. It was shown that there is a constant $\epsilon_{0}(n)>0$ depending only on $n$ such that there is an $F$-structure $\mathcal{F}$ of positive rank on a region covering $M_{\epsilon_{0}}$ in a Riemannian $n$-manifold $(M, g)$, where $M_{\epsilon_{0}}$ denotes a subset with injectivity radius $i_{g}(p)<\epsilon_{0}$ and sectional curvature sup $\left|K_{g}\right| \leq 1$, for any $p \in M_{\epsilon_{0}}$. See $[\mathbf{9}]$ and $[\mathbf{1 0}]$ for the definition of $F$-structure of positive rank.

2.2. A comparison theorem for calibrated submanifolds. In [26, Harvey and Lawson introduced the notion of a calibrated submanifold. If $(M, g)$ is a Riemannian manifold, and $\Theta$ is a closed $n$-form such that $\left.\Theta\right|_{\xi} \leq d v_{\xi}$ for any oriented $n$-plane $\xi$ in the tangent bundle of $M$, then $\Theta$ is called a calibration on $M$, where $d v_{\xi}$ denotes the volume form on $\xi$. An oriented $n$-submanifold $L$ of $M$ is called calibrated by the calibration $\Theta$, if $\left.\Theta\right|_{L}$ equals to the volume form of $\left.g\right|_{L}$ on $L$. Mclean studied the deformation theory of calibrated submanifolds in $\mathbf{3 3}$.

There are some examples of calibrated submanifolds: holomorphic submanifolds in Kähler manifolds, special Lagrangian submanifolds in CalabiYau manifolds, associative coassociative submanifolds in $G_{2}$-manifolds, Cayley submanifolds in $\operatorname{Spin}(7)$-manifolds (cf. [26, 28]) etc. If $(M, \omega, J, g)$ is a Kähler $m$-manifold, then $\frac{1}{n !} \omega^{n}, n \leq m$, are calibrations on $M$, and holomorphic $n$ submanifolds are calibrated by $\frac{1}{n !} \omega^{n}$. If $(M, \omega, J, g, \Omega)$ is a Ricci-flat CalabiYau $n$-manifold, then, for any $\theta \in \mathbb{R}, \operatorname{Re} e^{\sqrt{-1} \theta} \Omega$ is a calibration on $M$, and a special Lagrangian submanifold $L$ of phase $\theta$ is calibrated by $\operatorname{Re} e^{\sqrt{-1} \theta} \Omega$. If $(M, g)$ is a Riemannian manifold with holonomy group $G_{2}$, then $M$ admits a parallel 3 -form $\phi$, which is a calibration on $M$, and $*_{g} \phi$ is a calibration 
4-form on $M$. Submanifolds calibrated by $\phi$ are called associative submanifolds, and submanifolds calibrated by $*_{g} \phi$ are called coassociative submanifolds (cf. 28). If $(M, g)$ is a Riemannian manifold with holonomy group $\operatorname{Spin}(7)$, then $M$ admits a calibration 4 -form $\Omega$, and Cayley submanifolds are submanifolds calibrated by $\Omega$ (cf. [28]).

In [19], a volume comparison theorem for calibrated submanifolds was obtained.

Theorem 2.5 (Theorem 2.0.1. in $\mathbf{1 9})$ ). Let $(M, g)$ be a closed Riemannian manifold, $\Theta$ be a calibration $n$-form, and $p \in M$. Assume that the sectional curvature $K_{g}$ satisfies

$$
\sup _{B_{g}\left(p, \frac{2 \pi}{\sqrt{\Lambda}}\right)} K_{g} \leq \Lambda, \quad \Lambda>0
$$

and there is a submanifold $L$ calibrated by $\Theta$ such that $p \in L$. Then

$$
\operatorname{Vol}_{g}\left(B_{g}(p, r) \cap L\right) \geq \operatorname{Vol}_{h_{1}}\left(B_{h_{1}}(r)\right),
$$

for any $r \leq \min \left\{i_{g}(p), \frac{\pi}{\sqrt{\Lambda}}\right\}$, where $h_{1}$ denotes the standard metric on $S^{n}$ with constant curvature $\Lambda$, and $B_{h_{1}}(r)$ denotes a metric r-ball in $S^{n}$.

2.3. Implicit function theorem. For studying the deformation of special Lagrangian fibrations, we need the following quantitative version of implicit function theorem.

Theorem 2.6 (Theorem 3.2 in $[\mathbf{3 6})$ ). Let $\left(\mathfrak{B}_{1},\|\cdot\|_{1}\right)$ and $\left(\mathfrak{B}_{2},\|\cdot\|_{2}\right)$ be two Banach spaces, $\|\cdot\|_{E}$ be the standard Euclidean metric on $\mathbb{R}^{n}, U \subset \mathbb{R}^{n} \times \mathfrak{B}_{1}$ be an open set, and $\mathfrak{F}: U \longrightarrow \mathfrak{B}_{2}$ be a continuously differentiable map. Let

$$
D \mathfrak{F}(y, \sigma)(\dot{y}+\dot{\sigma})=D_{y} \mathfrak{F}(y, \sigma) \dot{y}+D_{\sigma} \mathfrak{F}(y, \sigma) \dot{\sigma},
$$

for $(y, \sigma) \in U, \dot{y} \in \mathbb{R}^{n}$ and $\dot{\sigma} \in \mathfrak{B}_{1}$. Assume that $(0,0) \in U$ and $D_{\sigma} \mathfrak{F}(0,0)$ : $\mathfrak{B}_{1} \longrightarrow \mathfrak{B}_{2}$ has a bounded linear inverse $D_{\sigma} \mathfrak{F}(0,0)^{-1}: \mathfrak{B}_{2} \longrightarrow \mathfrak{B}_{1}$ with

$$
\left\|D_{\sigma} \mathfrak{F}(0,0)^{-1}\right\| \leq \bar{C}
$$

for a constant $\bar{C}>0$. Let $r>0, \delta_{0}>\delta>0$ be such constants that if $\left\|y_{0}\right\|_{E}<r$ and $\|\sigma\|_{1} \leq \delta_{0}$, then $\left(y_{0}, \sigma\right) \in U$,

$$
\left\|D_{\sigma} \mathfrak{F}\left(y_{0}, \sigma\right)-D_{\sigma} \mathfrak{F}(0,0)\right\| \leq \frac{1}{2 \bar{C}} \text { and }\left\|\mathfrak{F}\left(y_{0}, 0\right)\right\|_{2} \leq \frac{\delta}{4 \bar{C}} .
$$

Then, for any $\|y\|_{E}<r$, there exists a unique $\sigma(y) \in \mathfrak{B}_{1}$ such that

$$
\mathfrak{F}(y, \sigma(y))=0, \quad\|\sigma(y)\|_{1} \leq \delta .
$$

Furthermore,

$$
D \sigma(y) \dot{y}=-D_{\sigma} \mathfrak{F}(y, \sigma)^{-1} D_{y} \mathfrak{F}(y, \sigma) \dot{y} .
$$


The difference between this version of implicit function theorem and the usual one (cf. $\left[\mathbf{2 4}\right.$ ) is that we use the condition $\|\mathfrak{F}(y, 0)\|_{2} \leq \frac{\delta}{4 \bar{C}}$ instead of the condition $\mathfrak{F}(0,0)=0$ in addition to other quantitative estimates.

3. The blow-up limit. Let $\left\{\left(M_{k}, \omega_{k}, J_{k}, g_{k}, \Omega_{k}\right)\right\}$ be a family of closed Ricci-flat Calabi-Yau $n$-manifolds with $\left[\omega_{k}\right] \in H^{2}\left(M_{k}, \mathbb{Z}\right)$, and $p_{k} \in M_{k}$. Assume that

i) the injectivity radius and the sectional curvature fulfil the following estimates:

$$
i_{g_{k}}\left(p_{k}\right)<\frac{1}{k}, \quad \sup _{B_{g_{k}}\left(p_{k}, 1\right)}\left|K_{g_{k}}\right| \leq 1,
$$

ii) there is a $\sigma \gg 1$ such that $\left[\left.\Omega_{k}\right|_{B_{g_{k}}\left(p_{k}, \sigma i_{g_{k}}\left(p_{k}\right)\right)}\right] \neq 0$ in $H^{n}\left(B_{g_{k}}\left(x_{k}, \sigma i_{g_{k}}\left(p_{k}\right)\right)\right.$, $\mathbb{C})$.

If we denote $\tilde{\omega}_{k}=i_{g_{k}}^{-2}\left(p_{k}\right) \omega_{k}, \tilde{g}_{k}=i_{g_{k}}^{-2}\left(p_{k}\right) g_{k}$, and $\tilde{\Omega}_{k}=i_{g_{k}}^{-n}\left(p_{k}\right) \Omega_{k}$, then

$$
i_{\tilde{g}_{k}}\left(p_{k}\right)=1, \quad \sup _{B_{\tilde{g}_{k}}\left(p_{k}, k\right)}\left|K_{\tilde{g}_{k}}\right| \leq \frac{1}{k^{2}},
$$

and $\left[\left.\tilde{\Omega}_{k}\right|_{B_{\tilde{g}_{k}}\left(p_{k}, \sigma\right)}\right] \neq 0$ in $H^{n}\left(B_{\tilde{g}_{k}}\left(p_{k}, \sigma\right), \mathbb{C}\right)$. By the Cheeger-Gromov's convergence theorem (cf. Theorem 2.4 , a subsequence of $\left(M_{k}, \tilde{\omega}_{k}, \tilde{g}_{k}, J_{k}, \tilde{\Omega}_{k}, p_{k}\right)$ converges to a complete flat Calabi-Yau $n$-manifold $\left(X, \omega_{0}, g_{0}, J_{0}, \Omega_{0}, p_{0}\right)$ in the $C^{\infty}$-sense, i.e. for any $r>\sigma$, there are embeddings $F_{r, k}: B_{g_{0}}\left(p_{0}, r\right) \longrightarrow M_{k}$ such that $F_{r, k}\left(p_{0}\right)=p_{k}$, and $F_{r, k}^{*} \tilde{g}_{k}$ (resp. $F_{r, k}^{*} \tilde{\omega}_{k}$ and $F_{r, k}^{*} \tilde{\Omega}_{k}$ ) converges to $g_{0}$ (resp. $\omega_{0}$ and $\left.\Omega_{0}\right)$ in the $C^{\infty}$-sense. The purpose of this section is to prove that $\left(X, \omega_{0}, \Omega_{0}\right)$ admits a special Lagrangian fibration.

By the smooth convergence, $i_{g_{0}}\left(p_{0}\right)=\lim _{k \rightarrow \infty} i_{\tilde{g}_{k}}\left(p_{k}\right)=1$. The soul theorem (cf. [10], [34]) implies that there is a compact flat totally geodesic submanifold $S \subset X$, the soul, such that $\left(X, g_{0}\right)$ is isometric to the total space of the normal bundle $\nu(S)$ with a metric induced by $\left.g_{0}\right|_{S}$ and a natural flat connection.

LEMMA 3.1. $\operatorname{dim}_{\mathbb{R}} S \geq n$.

Proof. If $\operatorname{dim}_{\mathbb{R}} S<n$, then

$$
H^{n}(X, \mathbb{C})=H^{n}\left(T_{r}(S), \mathbb{C}\right)=H^{n}(S, \mathbb{C})=\{0\}
$$

for any $r>0$, where $T_{r}(S)=\left\{p \in X \mid \operatorname{dist}_{g_{0}}(p, S) \leq r\right\}$. Let $r_{0}>r_{1}>\sigma$ be such that $T_{r_{1}}(S) \subset B_{g_{0}}\left(p_{0}, r_{0}\right)$, and $F_{r_{0}, k}\left(T_{r_{1}}(S)\right) \supset B_{\tilde{g}_{k}}\left(p_{k}, \sigma\right)$ for $k \gg 1$. Then the inclusion maps induce homeomorphisms on cohomology groups

$$
\begin{aligned}
H^{n}(M, \mathbb{C}) & \longrightarrow H^{n}\left(F_{r_{0}, k}\left(T_{r_{1}}(S)\right), \mathbb{C}\right) \longrightarrow H^{n}\left(B_{\tilde{g}_{k}}\left(p_{k}, \sigma\right), \mathbb{C}\right), \\
\text { and }\left[\tilde{\Omega}_{k}\right] & \mapsto\left[\left.\tilde{\Omega}_{k}\right|_{F_{r_{0}, k}\left(T_{r_{1}}(S)\right)}\right] \mapsto\left[\left.\tilde{\Omega}_{k}\right|_{B_{\tilde{g}_{k}}\left(p_{k}, \sigma\right)}\right] \neq 0 .
\end{aligned}
$$


Thus $\left[\left.\tilde{\Omega}_{k}\right|_{F_{r_{0}, k}\left(T_{r_{1}}(S)\right)}\right] \neq 0$ in $H^{n}\left(F_{r_{0}, k}\left(T_{r_{1}}(S)\right), \mathbb{C}\right)$, which contradicts to $H^{n}\left(F_{r_{0}, k}\left(T_{r_{1}}(S)\right), \mathbb{C}\right) \cong H^{n}\left(T_{r_{1}}(S), \mathbb{C}\right)=\{0\}$.

If $\pi_{h}: \tilde{S} \longrightarrow S$ is the holonomy covering of $S$, then by Bieberbach's theorem (cf. 10], 34$),\left(\tilde{S}, \pi_{h}^{*} g_{0}\right)$ is isometric to a flat torus and $\pi_{h}$ has finite order of at most $\lambda(n)$, for a constant $\lambda(n)$ depending on $n$ only. If we denote $\bar{\pi}: \mathbb{C}^{n} \longrightarrow X$ the universal covering of $X$ with $\bar{\pi}(0) \in S$, then $\bar{S}=\bar{\pi}^{-1}(S)$ is a real linear subspace of $\mathbb{C}^{n}$, and $\omega_{E}=\bar{\pi}^{*} \omega_{0}$ (resp. $\Omega_{E}=\bar{\pi}^{*} \Omega_{0}$ ) is the standard flat Kähler form (resp. the standard holomorphic volume form), i.e. $\omega_{E}=\sqrt{-1} \sum_{\alpha} d z_{\alpha} \wedge d \bar{z}_{\alpha}$ and $\Omega_{E}=d z_{1} \wedge \cdots \wedge d z_{n}$ under some coordinates $z_{1}, \cdots, z_{n}$ on $\mathbb{C}^{n}$. Note that there is a lattice $\Lambda \subset \bar{S}$ such that $\tilde{S}=\bar{S} / \Lambda$. If we denote $\mathfrak{q}: \bar{S} \longrightarrow \tilde{S}$ the quotient map, then $\bar{\pi}=\pi_{h} \circ \mathfrak{q}$.

Lemma 3.2. $\operatorname{dim}_{\mathbb{R}} \bar{S}=n$, and there is a constant $\theta_{0} \in \mathbb{R}$ such that $\left.\omega_{E}\right|_{\bar{S}}=0$ and $\left.\operatorname{Im} e^{\sqrt{-1} \theta_{0}} \Omega_{E}\right|_{\bar{S}}=0$. Moreover, $S$ is a special Lagrangian submanifold of phase $\theta_{0}$ in $\left(X, \omega_{0}, \Omega_{0}\right)$, i.e. $\operatorname{dim}_{\mathbb{R}} S=n$,

$$
\left.\omega_{0}\right|_{S} \equiv 0, \text { and }\left.\operatorname{Im} e^{\sqrt{-1} \theta_{0}} \Omega_{0}\right|_{S}=0 .
$$

Proof. If $\left.\omega_{E}\right|_{\bar{S}} \neq 0$ and thus $\left.\omega_{0}\right|_{S} \neq 0$, then there are two vectors $v_{1}, v_{2} \in$ $\bar{S}$ such that $\omega_{E}\left(v_{1}, v_{2}\right)>0$. By perturbing $v_{1}$ and $v_{2}$ a little bit if necessary, we conclude that $\tilde{\Sigma}=\mathfrak{q}\left(\left\{t_{1} v_{1}+t_{2} v_{2} \mid t_{i} \in \mathbb{R}\right\}\right)$ is a closed 2-torus in $\tilde{S}$, i.e. a closed 2-parameter subgroup. Thus $\Sigma=\pi_{h}(\tilde{\Sigma})$ is a closed oriented surface in $S$, which satisfies

$$
\int_{\Sigma} \omega_{0} \geq \frac{1}{\lambda(n)} \int_{\tilde{\Sigma}} \pi_{h}^{*} \omega_{0} \geq \frac{\omega_{E}\left(v_{1}, v_{2}\right)}{\lambda(n)\left\|v_{1} \wedge v_{2}\right\|_{h_{E}}} V_{E}>0,
$$

where $V_{E}$ denotes the Euclidean area of the intersection of $\left\{t_{1} v_{1}+t_{2} v_{2} \mid\right.$ $\left.t_{i} \in \mathbb{R}\right\}$ with the fundamental domain of the quotient map $\mathfrak{q}$. From the smooth convergence of $\left(M_{k}, \tilde{\omega}_{k}, \tilde{g}_{k}\right)$,

$$
\lim _{k \longrightarrow \infty} i_{g_{k}}^{-2}\left(p_{k}\right) \int_{F_{r, k}(\Sigma)} \omega_{k}=\lim _{k \longrightarrow \infty} \int_{F_{r, k}(\Sigma)} \tilde{\omega}_{k}=\lim _{k \longrightarrow \infty} \int_{\Sigma} F_{r, k}^{*} \tilde{\omega}_{k}=\int_{\Sigma} \omega_{0},
$$

for $r \gg 1$ such that $\Sigma \subset B_{g_{0}}\left(p_{0}, r\right)$. Thus

$$
0<\frac{1}{2} i_{g_{k}}^{2}\left(p_{k}\right) \int_{\Sigma} \omega_{0} \leq \int_{F_{r, k}(\Sigma)} \omega_{k} \leq 2 i_{g_{k}}^{2}\left(p_{k}\right) \int_{\Sigma} \omega_{0} \leq 2 k^{-2} \int_{\Sigma} \omega_{0}<1,
$$

for $k \gg 1$. Since $\left[F_{r, k}(\Sigma)\right] \in H_{2}\left(M_{k}, \mathbb{Z}\right)$ and $\left[\omega_{k}\right] \in H^{2}\left(M_{k}, \mathbb{Z}\right)$, we obtain

$$
\int_{F_{r, k}(\Sigma)} \omega_{k} \in \mathbb{Z}
$$

which is a contradiction. Hence $\left.\omega_{E}\right|_{\bar{S}} \equiv 0$ and $\left.\omega_{0}\right|_{S} \equiv 0$, which implies that $S$ is a Lagrangian submanifold $\left(X, \omega_{0}\right)$ by combining Lemma 3.1 . 
Since $\bar{S}$ is a Lagrangian linear subspace of $\left(\mathbb{C}^{n}, \omega_{E}\right)$, there is a $\theta_{0} \in \mathbb{R}$ such that $\left.\operatorname{Im} e^{\sqrt{-1} \theta_{0}} \Omega_{E}\right|_{\bar{S}}=0$. This implies that $\bar{S}$ is a special Lagrangian linear subspace of phase $\theta_{0}$ in $\left(\mathbb{C}^{n}, \omega_{E}, \Omega_{E}\right)$. Thus $S$ is a special Lagrangian submanifold of phase $\theta_{0}$ in $\left(X, \omega_{0}, \Omega_{0}\right)$, i.e.

$$
\left.\omega_{0}\right|_{S}=0,\left.\quad \operatorname{Im} e^{\sqrt{-1} \theta_{0}} \Omega_{0}\right|_{S}=0 .
$$

Lemma 3.3. For $k \gg 1,\left[\left.F_{r, k}^{*} \tilde{\omega}_{k}\right|_{S}\right]=0$ in $H^{2}(S, \mathbb{R})$.

Proof. By the smooth convergence of $\tilde{\omega}_{k}$ and Lemma 3.2 .

$$
\lim _{k \rightarrow \infty} \int_{F_{r, k}(A)} \tilde{\omega}_{k}=\int_{A} \omega_{0}=0,
$$

for any cycle $A \in H_{2}(S, \mathbb{Z})$. For $k \gg 1$, we have

$$
\left|\int_{F_{r, k}(A)} \omega_{k}\right|=i_{g_{k}}^{2}\left(p_{k}\right)\left|\int_{F_{r, k}(A)} \tilde{\omega}_{k}\right|<\frac{1}{2 k^{2}}<1 .
$$

Since $\left[F_{r, k}(A)\right] \in H_{2}\left(M_{k}, \mathbb{Z}\right)$ and $\left[\omega_{k}\right] \in H^{2}\left(M_{k}, \mathbb{Z}\right)$, we obtain $\int_{F_{r, k}(A)} \omega_{k} \in \mathbb{Z}$. This implies that $\left|\int_{F_{r, k}(A)} \omega_{k}\right|=0$, and we obtain the conclusion

$$
\int_{A} F_{r, k}^{*} \tilde{\omega}_{k}=0
$$

Let $\tilde{X}$ be the total space of the pull-back $\pi_{h}^{*} \nu(S)$ of the normal bundle. Note that we can identify the zero section of $\pi_{h}^{*} \nu(S)$ with $\tilde{S}$, and the covering $\pi_{h}$ extends to a finite covering $\pi: \tilde{X} \longrightarrow X$ of $X$, i.e. $\tilde{S}=\pi^{-1}(S) \subset \tilde{X}$, and $\left.\pi\right|_{\tilde{S}}=\pi_{h}$. The fundamental group $\pi_{1}(\tilde{S}) \cong \pi_{1}(\tilde{X})$ is isomorphic to the lattice $\Lambda, \pi_{1}(\tilde{X})$ is a normal subgroup of $\pi_{1}(X)=\pi_{1}(S)$, and the covering group $\Gamma \cong \pi_{1}(S) / \pi_{1}(\tilde{S})=\pi_{1}(X) / \pi_{1}(\tilde{X})$. Note that $\pi_{1}(\tilde{X})$ (resp. $\pi_{1}(X)$ ) acts on $\mathbb{C}^{n}$ preserving $g_{E}, \omega_{E}$ and $\Omega_{E}, \bar{S}$ is invariant, $\tilde{X}=\mathbb{C}^{n} / \pi_{1}(\tilde{X})$ (resp. $\left.X=\mathbb{C}^{n} / \pi_{1}(X)\right)$, and $\tilde{S}=\bar{S} / \pi_{1}(\tilde{S})=\bar{S} / \Lambda\left(\operatorname{resp} . S=\bar{S} / \pi_{1}(S)\right)$.

Proposition 3.4. Let $\bar{S}^{\perp}$ be the orthogonal complement of $\bar{S}$ in $\mathbb{C}^{n}$, i.e. $\mathbb{C}^{n}=\bar{S} \oplus \bar{S}^{\perp}$, and $g_{E}(v, w)=0$, for any $v \in \bar{S}$ and $w \in \bar{S}^{\perp}$. Then

i) $\left(\tilde{X}, \pi^{*} g_{0}\right)$ is isometric to $\left(T^{n} \times \bar{S}^{\perp}, h+h_{E}\right)$, where $T^{n}=\bar{S} / \Lambda=\tilde{S}$, $h_{E}=\left.g_{E}\right|_{\bar{S}^{\perp}}$, and $h$ is the standard flat metric on $T^{n}$ induced by $\left.g_{E}\right|_{\bar{S}}$.

ii) The action of $\Gamma$ on $\tilde{X}$ is a product action, i.e. there are $\Gamma$-actions on $T^{n}$ and $\bar{S}^{\perp}$ such that $\gamma \cdot(x, y)=(\gamma \cdot x, \gamma \cdot y)$ for any $\gamma \in \Gamma, x \in T^{n}$ and $y \in \bar{S}^{\perp}$. Furthermore, $T^{n} \times\{0\}$ is $\Gamma$-invariant, and $S=\pi\left(T^{n} \times\{0\}\right)=$ $\left(T^{n} \times\{0\}\right) / \Gamma$.

iii) $\left.\quad \pi^{*} \omega_{0}\right|_{T^{n} \times\{y\}} \equiv 0, \quad$ and $\left.\quad \pi^{*} \operatorname{Im} e^{\sqrt{-1} \theta_{0}} \Omega_{0}\right|_{T^{n} \times\{y\}} \equiv 0$, for any $y \in \bar{S}^{\perp}$, and a constant $\theta_{0} \in \mathbb{R}$. 
Proof. We choose coordinates $x_{1}, \cdots, x_{n}$ on $\bar{S}$ and $y_{1}, \cdots, y_{n}$ on $\bar{S}^{\perp}$ such that

$g_{E}=\sum\left(d x_{j}^{2}+d y_{j}^{2}\right), \quad \omega_{E}=\sum d x_{j} \wedge d y_{j}, \quad e^{\sqrt{-1} \theta_{0}} \Omega_{E}=\bigwedge_{j=1}^{n}\left(d x_{j}+\sqrt{-1} d y_{j}\right)$.

If $\mathcal{G}$ is a subgroup of the fundamental group $\pi_{1}(X)=\pi_{1}(S)$, then $\mathcal{G}$ acts on $\mathbb{C}^{n}$ preserving $g_{E}, \omega_{E}$ and $\Omega_{E}$, and $\bar{S}$ is a invariant subspace. For any $\gamma \in \mathcal{G}$, we have $\gamma \cdot(v+w)=G_{\gamma}(v+w)+b_{\gamma}$, where $G_{\gamma} \in U\left(\mathbb{C}^{n}\right), b_{\gamma} \in \bar{S}, v \in \bar{S}$ and $w \in \bar{S}^{\perp}$. Since $\bar{S}$ is invariant, we then obtain $G_{\gamma}(v+w)=A_{\gamma} v+B_{\gamma} w+C_{\gamma} w$ where $A_{\gamma} \in S O(\bar{S}), B_{\gamma} \in S O\left(\bar{S}^{\perp}\right)$, and $C_{\gamma} \in \operatorname{Hom}\left(\bar{S}^{\perp}, \bar{S}\right)$. Moreover, $G_{\gamma} \in S O\left(\mathbb{R}^{2 n}\right)$ implies $C_{\gamma}=0$. Since $\omega_{E}\left(G_{\gamma}(v+w), G_{\gamma}(v+w)\right)=\omega_{E}(v+w, v+w)$, we have $B_{\gamma}=A_{\gamma}^{-1, T}=A_{\gamma}$, and $\gamma \cdot(v+w)=A_{\gamma}(v+w)+b_{\gamma}$. Thus $\pi_{1}(\tilde{X}) \cong \Lambda$ acts on $\mathbb{C}^{n}$ given by $\gamma \cdot(v+w)=v+w+b_{\gamma}, b_{\gamma} \in \Lambda$, for any $v \in \bar{S}$ and $w \in \bar{S}^{\perp}$. This implies that $\tilde{X}=\mathbb{C}^{n} / \pi_{1}(\tilde{X}) \cong \bar{S} / \Lambda \times \bar{S}^{\perp}=\tilde{S} \times \bar{S}^{\perp}$, and $\pi^{*} g_{0}=h+h_{E}$ where $h_{E}=\left.g_{E}\right|_{\bar{S}^{\perp}}$, and $h$ is the standard flat metric on $\tilde{S}$ induced by $\left.g_{E}\right|_{\bar{S}}$.

The $\pi_{1}(X)$-action on $\mathbb{C}^{n}$ descents to a $\Gamma$-action on $\tilde{X}$, which is a product action since the $\pi_{1}(X)$-action is. Moreover, $\tilde{S} \times\{0\}$ is a invariant set as $\bar{S} \times\{0\}$ is invariant under the $\pi_{1}(X)$-action. If $\mathfrak{q}_{1}: \mathbb{C}^{n} \longrightarrow \mathbb{C}^{n} / \Lambda=\tilde{X}$ is the quotient map then $\bar{\pi}=\pi \circ \mathfrak{q}_{1}, g_{E}=\mathfrak{q}_{1}^{*} \pi^{*} g_{0}, \omega_{E}=\mathfrak{q}_{1}^{*} \pi^{*} \omega_{0}$, and $\Omega_{E}=\mathfrak{q}_{1}^{*} \pi^{*} \Omega_{0}$. Since $\left.\omega_{E}\right|_{\bar{S} \times\{y\}}=0$ and $\left.e^{\sqrt{-1} \theta_{0}} \Omega_{E}\right|_{\bar{S} \times\{y\}}=0$ for $y \in \bar{S}^{\perp}$, we obtain

$$
\left.\pi^{*} \omega_{0}\right|_{T^{n} \times\{y\}} \equiv 0, \text { and }\left.\pi^{*} \operatorname{Im} e^{\sqrt{-1} \theta_{0}} \Omega_{0}\right|_{T^{n} \times\{y\}} \equiv 0,
$$

for a constant $\theta_{0} \in \mathbb{R}$.

REMARK 3.5. The coordinates $x_{1}, \cdots, x_{n}$ on $\bar{S}$ in the proof above induce parallel 1-forms $d x_{1}, \cdots, d x_{n}$ on $(\tilde{S}, h)$ which are pointwise linear independent, i.e. $d x_{1}, \cdots, d x_{n}$ is a global parallel frame field. Under the coordinates $y_{1}, \cdots, y_{n}$ on $\bar{S}^{\perp}$, we have these formulas

$$
\pi^{*} g_{0}=\sum\left(d x_{j}^{2}+d y_{j}^{2}\right), \quad \pi^{*} \omega_{0}=\sum d x_{j} \wedge d y_{j}, \quad e^{\sqrt{-1} \theta_{0}} \pi^{*} \Omega_{0}=\bigwedge_{j=1}^{n}\left(d x_{j}+\sqrt{-1} d y_{j}\right)
$$

REMARK 3.6. The natural projection $f_{0}: \tilde{X} \longrightarrow \bar{S}^{\perp}$ is equivariant under the $\Gamma$ actions on $\tilde{X}$ and $\bar{S}^{\perp}$. For any $y \in \bar{S}^{\perp}, f_{0}^{-1}(y)=\tilde{S} \times\{y\}$ and $f_{0}$ is a special Lagrangian fibration on $\left(\tilde{X}, \pi^{*} \omega_{0}, e^{\sqrt{-1} \theta_{0}} \pi^{*} \Omega_{0}\right)$, i.e. $\operatorname{dim}_{\mathbb{R}} f_{0}^{-1}(y)=n$,

$$
\left.\pi^{*} \omega_{0}\right|_{f_{0}^{-1}(y)} \equiv 0,\left.\quad e^{\sqrt{-1} \theta_{0}} \pi^{*} \Omega_{0}\right|_{f_{0}^{-1}(y)} \equiv 0 .
$$

4. Local special Lagrangian fibrations. In this section, we study the deformation of special Lagrangian fibrations under the convergence of CalabiYau metrics. Let $(Y, \omega, g, J, \Omega)$ be a complete flat Calabi-Yau $n$-manifold. 
Condition 4.1. Assume that

i) $Y=T^{n} \times \mathbb{R}^{n}, g=h+h_{E}$, and the natural projection $f: Y \longrightarrow \mathbb{R}^{n}$ is a special Lagrangian fibration of $(Y, \omega, \Omega)$, where $T^{n}=\mathbb{R}^{n} / \Lambda$ is a torus, $\Lambda$ is a lattice in $\mathbb{R}^{n}, h_{E}$ is the standard Euclidean metric on $\mathbb{R}^{n}$, and $h$ is the standard flat metric induced by $h_{E}$.

ii) There are parallel 1-forms $d x_{1}, \cdots, d x_{n}$ on $\left(T^{n}, h\right)$, which are pointwise linear independent, and coordinates $y_{1}, \cdots, y_{n}$ on $\mathbb{R}^{n}$ such that

$$
g=h+h_{E}=\sum\left(d x_{j}^{2}+d y_{j}^{2}\right), \quad \omega=\sum d x_{j} \wedge d y_{j}, \quad \Omega=\bigwedge_{j=1}^{n}\left(d x_{j}+\sqrt{-1} d y_{j}\right) .
$$

iii) There is a family of Calabi-Yau structures $\left(\omega_{k}, g_{k}, J_{k}, \Omega_{k}\right)$ converging to $(\omega, g, J, \Omega)$ in the $C^{\infty}$-sense on $Y_{2 r}=T^{n} \times B_{h_{E}}(0,2 r)$ for a $r \gg 1$, where $B_{h_{E}}(0,2 r)=\left\{y \in \mathbb{R}^{n} \mid\|y\|_{h_{E}}<2 r\right\}$. Moreover, $\omega_{k} \in[\omega] \in H^{2}\left(Y_{2 r}, \mathbb{R}\right)$.

vi) There is a finite group $\Gamma$ acting on $Y_{2 r}$ preserving $\omega_{k}, g_{k}, \Omega_{k}, \omega, g, \Omega$, and $T^{n} \times\{0\}$ is a invariant set. The $\Gamma$-action is a product action on $T^{n} \times B_{h_{E}}(0,2 r)$. The natural projection $f: Y \longrightarrow \mathbb{R}^{n}$ is $\Gamma$-equivariant.

The goal of this section is to construct equivariant special Lagrangian fibrations on $\left(Y_{r}, \omega_{k}, \Omega_{k}\right)$ for $k \gg 1$.

Denote $L=T^{n} \times\{0\}$, which is a special Lagrangian submanifold of $(Y, \omega, \Omega)$, i.e. $\left.\omega\right|_{L}=0$ and $\left.\operatorname{Im} \Omega\right|_{L}=0$. Note that we can identify $Y$ with the total space of the normal bundle $\nu(L)$ by the exponential map from $\nu(L)$ to $Y$, $\exp _{L, g}:\left(x, \sum_{j} y_{j} \frac{\partial}{\partial y_{j}}\right) \mapsto(x, y)$ where $x \in L$ and $y=\left(y_{1}, \cdots, y_{n}\right)$. There is a canonical bundle isomorphism from $\nu(L)$ to the cotangent bundle $T^{*} L$ given by $v \mapsto \iota(v) \omega$ where $v \in \nu_{x}(L)$. Thus we can identify $Y$ with the total space of $T^{*} L$ by the map

$$
(x, y) \mapsto\left(x, \iota\left(\sum_{j} y_{j} \frac{\partial}{\partial y_{j}}\right) \omega\right)=\left(x, \sum_{j} y_{j} d x_{j}\right),
$$

where $x \in L$ and $y=\left(y_{1}, \cdots, y_{n}\right) \in \mathbb{R}^{n}$. We do not distinguish $Y$ from $T^{*} L$ in this section for convenience. For a 1 -form $\sigma$ on $L$, and a $y \in \mathbb{R}^{n}$, which can be regarded as a 1 -form from above,

$$
L(y, \sigma)=\{(x, y+\sigma(x)) \mid x \in L\}
$$

denotes the graph of $y+\sigma$, i.e. $y=\sum y_{j} d x_{j}, \sigma=\sum \sigma_{j} d x_{j}$, and

$$
L(y, \sigma)=\left\{\left(x, y_{1}+\sigma_{1}(x), \cdots, y_{n}+\sigma_{n}(x)\right) \mid x \in L\right\} .
$$

There are two constants $a_{k}>0$ and $\theta_{k} \in \mathbb{R}$, for any $k$, such that

$$
\int_{L} \Omega_{k}=a_{k} e^{-\sqrt{-1} \theta_{k}} \int_{L} \Omega_{0}=a_{k} e^{-\sqrt{-1} \theta_{k}} \int_{L} \operatorname{Re} \Omega_{0}
$$


$\lim _{k \longrightarrow \infty} a_{k}=1$ and $\lim _{k \longrightarrow \infty} \theta_{k}=0$ by the smooth convergence of $\Omega_{k}$. There are real 1-forms $\alpha_{k}$ and complex value $(n-1)$-forms $\beta_{k}$ such that

$$
\omega_{k}=\omega_{0}-d \alpha_{k}, \quad \Omega_{k}=a_{k} e^{-\sqrt{-1} \theta_{k}}\left(\Omega_{0}+d \beta_{k}\right)
$$

by $\omega_{k} \in[\omega] \in H^{2}\left(Y_{2 r}, \mathbb{R}\right)$. By the smooth convergence of $\omega_{k}$ and $\Omega_{k}$,

$$
\lim _{k \longrightarrow \infty}\left\|d \alpha_{k}\right\|_{C^{2}\left(Y_{2 r}, g\right)}=\lim _{k \rightarrow \infty}\left\|d \beta_{k}\right\|_{C^{2}\left(Y_{2 r}, g\right)}=0 .
$$

Define a diffeomorphism $\Pi: L \longrightarrow L(y, \sigma)$ by $x \mapsto(x, y+\sigma(x))$ for a $y \in \mathbb{R}^{n}$ and a 1 -form $\sigma$ on $L$. If

$$
\mathfrak{F}_{k}(y, \sigma)=\left(-\left.\Pi^{*} \omega_{k}\right|_{L(y, \sigma)},\left.{ }^{\prime} a_{k}^{-1} \Pi^{*} \operatorname{Im} e^{\sqrt{-1} \theta_{k}} \Omega_{k}\right|_{L(y, \sigma)}\right),
$$

where $*_{h}$ is the Hodge star operator on $(L, h)$, then $L(y, \sigma)$ is a special Lagrangian submanifold of $\left(Y, \omega_{k}, \Omega_{k}\right)$ of phase $\theta_{k}$ if and only if

$$
\mathfrak{F}_{k}(y, \sigma)=0 .
$$

A straightforward calculation (cf. 33 ) gives

$$
\mathfrak{F}_{k}(y, \sigma)=\left(d \sigma+\left.\Pi^{*} d \alpha_{k}\right|_{L(y, \sigma)}, *_{h} d *_{h} \sigma+\left.*_{h} \Pi^{*} d \operatorname{Im} \beta_{k}\right|_{L(y, \sigma)}\right) .
$$

We denote by $\Omega^{j}(L)$ the space of $j$-forms on $L$, and define two Banach spaces $\mathfrak{B}_{1}=C^{1, \alpha}\left(d \Omega^{0}(L) \oplus d^{* h} \Omega^{2}(L)\right)$ and $\mathfrak{B}_{2}=C^{0, \alpha}\left(d \Omega^{1}(L) \oplus d^{* h} \Omega^{1}(L)\right)$. Then $\mathfrak{F}_{k}$ defines a smooth map $\mathfrak{F}_{k}: \mathcal{U}(r) \longrightarrow \mathfrak{B}_{2}$ for any $k$, where $\mathcal{U}(r)=$ $\left\{\|y\|_{h_{E}}+\|\sigma\|_{C^{1, \alpha}(L, h)}<2 r \mid(y, \sigma) \in \mathbb{R}^{n} \times \mathfrak{B}_{1}\right\}$.

Lemma 4.2. For any $y \in B_{h_{E}}(0,2 r)$,

$$
\left\|\mathfrak{F}_{k}(y, 0)\right\|_{C^{0, \alpha}(L, h)} \leq C\left\|\left(d \alpha_{k}, d \beta_{k}\right)\right\|_{C^{1, \alpha}\left(Y_{2 r}, g\right)},
$$

for a constant $C$ independent of $k$.

Proof. Since

$$
\mathfrak{F}_{k}(y, 0)=\left(\left.\Pi^{*} d \alpha_{k}\right|_{L(y, 0)},\left.*_{h} \Pi^{*} d \operatorname{Im} \beta_{k}\right|_{L(y, 0)}\right),
$$

we obtain the conclusion by straightforward calculations.

The differentials of $\mathfrak{F}_{k}(y, \sigma)$ are

(5)

$D_{\sigma} \mathfrak{F}_{k}(y, \sigma) \dot{\sigma}=\left(d \dot{\sigma}, *_{h} d *_{h} \dot{\sigma}\right)+\left(D_{\sigma}\left(\left.\Pi^{*} d \alpha_{k}\right|_{L(y, \sigma)}\right) \dot{\sigma}, *_{h} D_{\sigma}\left(\left.\Pi^{*} d \operatorname{Im} \beta_{k}\right|_{L(y, \sigma)}\right) \dot{\sigma}\right)$,

$$
\begin{gathered}
D_{y} \mathfrak{F}_{k}(y, \sigma) \dot{y}=\left(D_{y}\left(\left.\Pi^{*} d \alpha_{k}\right|_{L(y, \sigma)}\right) \dot{y},{ }_{h} D_{y}\left(\left.\Pi^{*} d \operatorname{Im} \beta_{k}\right|_{L(y, \sigma)}\right) \dot{y}\right) \\
\text { and } D \mathfrak{F}_{k}(y, \sigma)(\dot{y}+\dot{\sigma})=D_{\sigma} \mathfrak{F}_{k}(y, \sigma) \dot{\sigma}+D_{y} \mathfrak{F}_{k}(y, \sigma) \dot{y} .
\end{gathered}
$$

Under the frame field $d x_{1}, \cdots, d x_{n}$ and coordinates $y_{1}, \cdots, y_{n}$,

$$
d \alpha_{k}=\sum_{i j}\left(\alpha_{k, i j} d x_{i} \wedge d x_{j}+\alpha_{k, i(n+j)} d x_{i} \wedge d y_{j}+\alpha_{k,(n+i)(n+j)} d y_{i} \wedge d y_{j}\right) .
$$


The differential is

$$
\begin{aligned}
D\left(\left.\Pi^{*} d \alpha_{k}\right|_{L(y, \sigma)}\right)(\dot{y}+\dot{\sigma}) & =\sum_{i j l}\left(\frac{\partial \alpha_{k, i j}}{\partial y_{l}}\left(\dot{y}_{l}+\dot{\sigma}_{l}\right) d x_{i} \wedge d x_{j}+\alpha_{k, i(n+j)} d x_{i} \wedge d \dot{\sigma}_{j}\right. \\
& +\frac{\partial \alpha_{k, i(n+j)}}{\partial y_{l}}\left(\dot{y}_{l}+\dot{\sigma}_{l}\right) d x_{i} \wedge d \sigma_{j}+\alpha_{k,(n+i)(n+j)} d \sigma_{i} \wedge d \dot{\sigma}_{j} \\
& \left.+\frac{\partial \alpha_{k,(n+i)(n+j)}}{\partial y_{l}}\left(\dot{y}_{l}+\dot{\sigma}_{l}\right) d \sigma_{i} \wedge d \sigma_{j}\right)
\end{aligned}
$$

We obtain

$$
\begin{aligned}
\left\|D_{\sigma}\left(\left.d \alpha_{k}\right|_{L}\right) \dot{\sigma}\right\|_{C^{0, \alpha}(L, h)} \leq & C\left\|d \alpha_{k}\right\|_{C^{1, \alpha}\left(Y_{2 r}, g\right)}\|\dot{\sigma}\|_{C^{1, \alpha}(L, h)}, \\
\left\|D_{\sigma}\left(\left.\Pi^{*} d \alpha_{k}\right|_{L(y, \sigma)}\right) \dot{\sigma}\right\|_{C^{0, \alpha}(L, h) \leq} & C\left\|d \alpha_{k}\right\|_{C^{1, \alpha}\left(Y_{2 r}, g\right)} \\
& \cdot\left(\sum_{l=0,1,2}\|\sigma\|_{C^{1, \alpha}(L, h)}^{l}\right)\|\dot{\sigma}\|_{C^{1, \alpha}(L, h)}, \\
\left\|D_{y}\left(\left.\Pi^{*} d \alpha_{k}\right|_{L(y, \sigma)}\right) \dot{y}\right\|_{C^{0, \alpha}(L, h) \leq} & C\left\|d \alpha_{k}\right\|_{C^{1, \alpha}\left(Y_{2 r}, g\right)} \\
& \cdot\left(\sum_{l=0,1,2}\|\sigma\|_{C^{1, \alpha}(L, h)}^{l}\right)\|\dot{y}\|_{h_{E}},
\end{aligned}
$$

for a constant $C$ independent of $k$. The same argument gives

$$
\begin{aligned}
\left\|D_{\sigma}\left(\left.d \operatorname{Im} \beta_{k}\right|_{L}\right) \dot{\sigma}\right\|_{C^{0, \alpha}(L, h) \leq} & C\left\|d \beta_{k}\right\|_{C^{1, \alpha}\left(Y_{2 r}, g\right)}\|\dot{\sigma}\|_{C^{1, \alpha}(L, h)}, \\
\left\|D_{\sigma}\left(\left.\Pi^{*} d \operatorname{Im} \beta_{k}\right|_{L(y, \sigma)}\right) \dot{\sigma}\right\|_{C^{0, \alpha}(L, h) \leq} & C\left\|d \beta_{k}\right\|_{C^{1, \alpha}\left(Y_{2 r}, g\right)} \\
& \cdot\left(\sum_{l=0,1, \cdots, n}\|\sigma\|_{C^{1, \alpha}(L, h)}^{l}\right)\|\dot{\sigma}\|_{C^{1, \alpha}(L, h)}, \\
\left\|D_{y}\left(\left.\Pi^{*} d \operatorname{Im} \beta_{k}\right|_{L(y, \sigma)}\right) \dot{y}\right\|_{C^{0, \alpha}(L, h) \leq} & C\left\|d \beta_{k}\right\|_{C^{1, \alpha}\left(Y_{2 r}, g\right)} \\
& \cdot\left(\sum_{l=0,1, \cdots, n}\|\sigma\|_{C^{1, \alpha}(L, h)}^{l}\right)\|\dot{y}\|_{h_{E}} .
\end{aligned}
$$

Lemma 4.3. The operator $D_{\sigma} \mathfrak{F}_{k}(0,0)$ is invertible for $k \gg 1$, and

$$
\left\|D_{\sigma} \mathfrak{F}_{k}(0,0)^{-1}\right\| \leq \bar{C},
$$

for a constant $\bar{C}>0$ independent of $k$.

Proof. Note that

$D_{\sigma} \mathfrak{F}_{k}(0,0) \dot{\sigma}=\left(d \dot{\sigma}, *_{h} d *_{h} \dot{\sigma}\right)+\left(D_{\sigma}\left(\left.d \alpha_{k}\right|_{L}\right) \dot{\sigma}, *_{h} D_{\sigma}\left(\left.d \operatorname{Im} \beta_{k}\right|_{L}\right) \dot{\sigma}\right)=\left(\mathcal{D}+V_{k}\right) \dot{\sigma}$, where $\mathcal{D}=d-*_{h} d *_{h}$ is the restriction of the Hodge Dirac operator $d+d^{* h}$ to the space of 1-forms, and, thus, is an elliptic operator of order 1. The Kernel Ker $\mathcal{D}$ of $\mathcal{D}$ is the space of harmonic 1 -forms, and therefore is orthogonal to $\mathfrak{B}_{1}$ with respect to the $L^{2}$-norm by the Hodge decomposition. Hence $\mathcal{D}: \mathfrak{B}_{1} \rightarrow \mathfrak{B}_{2}$ 
is injective, and by the standard elliptic estimate (cf. Proposition 1.5.2 in $\mathbf{2 8}$ and $[\mathbf{2 4}]$ ), we have

$$
\|\xi\|_{C^{1, \alpha}(L, h)} \leq C_{S}\|\mathcal{D} \xi\|_{C^{0, \alpha}(L, h)},
$$

for any $\xi \in \mathfrak{B}_{1}$, and a constant $C_{S}$ independent of $k$. From the definition of $\mathfrak{B}_{2}, \mathcal{D}$ is also surjective, which implies that $\mathcal{D}$ is an invertible operator from $\mathfrak{B}_{1}$ to $\mathfrak{B}_{2}$. Moreover,

$$
\left\|\mathcal{D}^{-1}\right\| \leq C_{S}
$$

By (7) and (8),

$$
\left\|V_{k}\right\| \leq C\left\|\left(d \alpha_{k}, d \beta_{k}\right)\right\|_{C^{1, \alpha}\left(Y_{2 r}, g\right)}<\frac{1}{2 C_{S}},
$$

for $k \gg 1$, and, thus,

$$
\left\|\mathcal{D}^{-1} V_{k}\right\|<\frac{1}{2}
$$

By the standard operator's theory (cf. $[40]), D_{\sigma} \mathfrak{F}_{k}(0,0)=\mathcal{D}+V_{k}$ is invertible, and the inverse operator is defined by

$$
D_{\sigma} \mathfrak{F}_{k}(0,0)^{-1}=\left(\sum_{j=0}^{\infty}(-1)^{j}\left(\mathcal{D}^{-1} V_{k}\right)^{j}\right) \mathcal{D}^{-1} .
$$

We obtain

$$
\left\|D_{\sigma} \mathfrak{F}_{k}(0,0)^{-1}\right\| \leq\left(\sum_{j=0}^{\infty} 2^{-j}\right)\left\|\mathcal{D}^{-1}\right\| \leq \bar{C},
$$

for a constant $\bar{C}>0$ independent of $k$.

Lemma 4.4. For any $\delta_{0} \ll 1$, there is a constant $k_{0} \gg 1$ such that, if $\|y\|_{h_{E}} \leq \frac{3 r}{2}$ and $\|\sigma\|_{C^{1, \alpha}(L, h)} \leq \delta_{0}$, and $k>k_{0}$, then

$$
\left\|D_{\sigma} \mathfrak{F}_{k}(y, \sigma)-D_{\sigma} \mathfrak{F}_{k}(0,0)\right\| \leq \frac{1}{2 \bar{C}} .
$$

Furthermore, also $D_{\sigma} \mathfrak{F}_{k}(y, \sigma)$ is invertible, and

$$
\left\|D_{\sigma} \mathfrak{F}_{k}(y, \sigma)^{-1}\right\| \leq 2 \bar{C} .
$$

Proof. By (5),

$$
\begin{aligned}
\left(D_{\sigma} \mathfrak{F}_{k}(y, \sigma)-D_{\sigma} \mathfrak{F}_{k}(0,0)\right) \dot{\sigma} & =\left(\left(D_{\sigma}\left(\left.\Pi^{*} d \alpha_{k}\right|_{L(y, \sigma)}\right)-D_{\sigma}\left(\left.d \alpha_{k}\right|_{L}\right)\right) \dot{\sigma},\right. \\
& \left.*_{h}\left(D_{\sigma}\left(\left.\Pi^{*} d \operatorname{Im} \beta_{k}\right|_{L(y, \sigma)}\right)-D_{\sigma}\left(\left.d \operatorname{Im} \beta_{k}\right|_{L}\right)\right) \dot{\sigma}\right) .
\end{aligned}
$$


We can take a $k_{0} \gg 1$ such that, for $k>k_{0}$,

$$
\begin{aligned}
\left\|D_{\sigma} \mathfrak{F}_{k}(y, \sigma)-D_{\sigma} \mathfrak{F}_{k}(0,0)\right\| & \leq 2 C\left\|\left(d \alpha_{k}, d \beta_{k}\right)\right\|_{C^{1, \alpha}\left(Y_{2 r}, g\right)}\left(\sum_{l=0,1, \cdots, n}\|\sigma\|_{C^{1, \alpha}(L, h)}^{l}\right) \\
& \leq 2 C\left\|\left(d \alpha_{k}, d \beta_{k}\right)\right\|_{C^{1, \alpha}\left(Y_{2 r}, g\right)} n \delta_{0} \\
& \leq \frac{1}{4 \bar{C}}
\end{aligned}
$$

by (2), (7) and (8). We obtain the first of the required estimates.

Note that $D_{\sigma} \mathfrak{F}_{k}(y, \sigma)=D_{\sigma} \mathfrak{F}_{k}(0,0)+\left(D_{\sigma} \mathfrak{F}_{k}(y, \sigma)-D_{\sigma} \mathfrak{F}_{k}(0,0)\right), D_{\sigma} \mathfrak{F}_{k}(0,0)$ is invertible, and $\left\|D_{\sigma} \mathfrak{F}_{k}(0,0)^{-1}\right\| \leq \bar{C}$. By the same arguments as in the proof of Lemma 4.3, and

$$
\left\|D_{\sigma} \mathfrak{F}_{k}(0,0)^{-1}\left(D_{\sigma} \mathfrak{F}_{k}(y, \sigma)-D_{\sigma} \mathfrak{F}_{k}(0,0)\right)\right\| \leq \frac{1}{2},
$$

also $D_{\sigma} \mathfrak{F}_{k}(y, \sigma)$ is invertible, and

$$
\left\|D_{\sigma} \mathfrak{F}_{k}(y, \sigma)^{-1}\right\| \leq\left(\sum_{j=0}^{\infty} 2^{-j}\right)\left\|D_{\sigma} \mathfrak{F}_{k}(0,0)^{-1}\right\| \leq 2 \bar{C} .
$$

Lemma 4.5. For a fixed $\delta<\delta_{0}$, there is a $k_{1}>k_{0}$ such that, for any $y \in B_{h_{E}}\left(0, \frac{3 r}{2}\right)$ and $k>k_{1}$, there is a unique $\sigma_{k}(y) \in \mathfrak{B}_{1}$, such that

$$
\mathfrak{F}_{k}\left(y, \sigma_{k}(y)\right)=0, \quad\left\|\sigma_{k}(y)\right\|_{C^{1, \alpha}(L, h)} \leq \delta,
$$

which implies that $L\left(y, \sigma_{k}(y)\right)$ is a special Lagrangian submanifold of $\left(Y_{2 r}, \omega_{k}, \Omega_{k}\right)$. Furthermore,

$$
\left\|D \sigma_{k}(y)\right\| \leq 2 n \delta \bar{C} C\left\|\left(d \alpha_{k}, d \beta_{k}\right)\right\|_{C^{1, \alpha}\left(Y_{2 r}, g\right)},
$$

for a constant $C$ independent of $k$.

Proof. Fix a $\delta<\delta_{0}$, there is a $k_{1}>k_{0}$ such that, for $k>k_{1}$, and any $y \in B_{h_{E}}(0,2 r)$,

$$
\left\|\mathfrak{F}_{k}(y, 0)\right\|_{C^{0, \alpha}(L, h)} \leq \frac{\delta}{4 \bar{C}}
$$

by Lemma 4.2 . By Theorem 2.6, Lemma 4.3 and Lemma 4.4 . for any $y \in$ $B_{h_{E}}\left(0, \frac{3 r}{2}\right)$ and $k>k_{1}$, there is a unique $\sigma_{k}(y) \in \mathfrak{B}_{1}$ such that

$$
\mathfrak{F}_{k}\left(y, \sigma_{k}(y)\right)=0, \quad\left\|\sigma_{k}(y)\right\|_{C^{1, \alpha}(L, h)} \leq \delta,
$$

which implies that $L\left(y, \sigma_{k}(y)\right)$ is a special Lagrangian submanifold of $\left(Y_{2 r}, \omega_{k}, \Omega_{k}\right)$. 
By (6), (7) and (8),

$$
\begin{aligned}
\left\|D_{y} \mathfrak{F}_{k}\left(y, \sigma_{k}\right)\right\| & \leq C\left\|\left(d \alpha_{k}, d \beta_{k}\right)\right\|_{C^{1, \alpha}\left(Y_{2 r}, g\right)}\left(\sum_{l=0,1, \cdots, n}\left\|\sigma_{k}\right\|_{C^{1, \alpha}(L, h)}^{l}\right) \\
& \leq C\left\|\left(d \alpha_{k}, d \beta_{k}\right)\right\|_{C^{1, \alpha}\left(Y_{2 r}, g\right)} n \delta
\end{aligned}
$$

for a constant $C$ independent of $k$. By Theorem 2.6 ,

$$
D \sigma_{k}(y) \dot{y}=-D_{\sigma} \mathfrak{F}_{k}\left(y, \sigma_{k}\right)^{-1} D_{y} \mathfrak{F}_{k}\left(y, \sigma_{k}\right) \dot{y} .
$$

We obtain the conclusion from Lemma 4.4 .

Proposition 4.6. For $k \gg 1$, there is an open set $W_{k}$ such that $Y_{2 r} \supset$ $W_{k} \supset Y_{r}$ and $\left(W_{k}, \omega_{k}, \Omega_{k}\right)$ admits an equivariant special Lagrangian fibration $f_{k}: W_{k} \longrightarrow B_{k}$ of phase $\theta_{k}$ over $B_{k} \subset \mathbb{R}^{n}$, i.e. there is a $\Gamma$-action on $B_{k}, f_{k}$ is a $\Gamma$-equivariant map, and $f_{k}$ is a special Lagrangian fibration of phase $\theta_{k}$, i.e.

$$
\left.\omega_{k}\right|_{f_{k}^{-1}(b)} \equiv 0,\left.\quad \operatorname{Im} e^{\sqrt{-1} \theta_{k}} \Omega_{k}\right|_{f_{k}^{-1}(b)} \equiv 0
$$

for any $b \in B_{k}$.

Proof. By Lemma 4.5, there is a unique $C^{1}$-map

$$
\sigma_{k}: B_{h_{E}}\left(0, \frac{3 r}{2}\right) \longrightarrow C^{1, \alpha}\left(d \Omega^{0}\left(T^{n}\right) \oplus d^{* h} \Omega^{2}\left(T^{n}\right)\right), \quad \text { by } y \mapsto \sigma_{k}(y),
$$

which satisfies

$$
\begin{aligned}
& \mathfrak{F}_{k}\left(y, \sigma_{k}(y)\right)=0, \quad\left\|\sigma_{k}(y)\right\|_{C^{1, \alpha}(L, h)} \leq \delta \ll 1, \\
& \text { and } \quad\left\|D \sigma_{k}(y)\right\| \leq 2 n \delta \bar{C} C\left\|\left(d \alpha_{k}, d \beta_{k}\right)\right\|_{C^{1, \alpha}\left(Y_{2 r}, g\right)} .
\end{aligned}
$$

This implies

$$
\left|\frac{\partial \sigma_{k, j}(y)}{\partial y_{i}}\right| \leq 2 n \delta \bar{C} C\left\|\left(d \alpha_{k}, d \beta_{k}\right)\right\|_{C^{1, \alpha}\left(Y_{2 r}, g\right)} \ll 1,
$$

for $k \gg k_{1}>1$.

Define a map $\Psi_{k}: Y_{\frac{3}{2} r} \longrightarrow Y_{2 r}$ by

$$
\Psi_{k}:(x, y) \mapsto\left(x, y_{1}+\sigma_{k, 1}(y), \cdots, y_{n}+\sigma_{k, n}(y)\right)=\left(x, y+\sigma_{k}(y)\right) .
$$

Note that the frame field $d x_{1}, \cdots, d x_{n}$ induces local coordinates $x_{1}, \cdots, x_{n}$ around any point on $L$, and the differential can be expressed as

$$
d \Psi_{k}:(\dot{x}, \dot{y}) \mapsto\left(\dot{x}_{j}+\sum \frac{\partial \sigma_{k, j}(y)}{\partial x_{i}} \dot{x}_{i}, \dot{y}_{j}+\sum \frac{\partial \sigma_{k, j}(y)}{\partial y_{i}} \dot{y}_{i}\right)
$$


under such local coordinates. Thus $d \Psi_{k}$ is an isomorphism when $k \gg 1$, which implies that $\Psi_{k}$ is an immersion. Furthermore, for $y_{1} \neq y_{2} \in \mathbb{R}^{n}$,

$$
\begin{aligned}
\Psi_{k}\left(x, y_{2}\right)-\Psi_{k}\left(x, y_{1}\right) & =\left(x, \cdots, \int_{0}^{1}\left(1+\frac{\partial \sigma_{k, j}\left((1-t) y_{2}+t y_{1}\right)}{\partial y_{j}} d t\right)\left(y_{2, j}-y_{1, j}\right), \cdots\right) \\
& \neq 0 .
\end{aligned}
$$

Hence $\Psi_{k}$ is an embedding.

Note that the $\Gamma$-action on $Y_{2 r}=T^{n} \times B_{h_{E}}(0,2 r)$ preserves $\omega_{k}, g_{k}, \Omega_{k}, \omega, g, \Omega$, and is a product action on $T^{n} \times B_{h_{E}}(0,2 r)$, i.e. there are $\Gamma$-actions on $T^{n}$ and $B_{h_{E}}(0,2 r)$ such that $\gamma \cdot(x, y)=(\gamma \cdot x, \gamma \cdot y)$ for any $\gamma \in \Gamma, x \in T^{n}$, and $y \in B_{h_{E}}(0,2 r)$. Under identification map (1),

$$
\begin{aligned}
(\gamma \cdot x, \gamma \cdot y)=\left(\gamma \cdot x, \iota\left(\gamma_{*} \sum_{j} y_{j} \frac{\partial}{\partial y_{j}}\right) \omega\right) & =\left(\gamma \cdot x, \gamma^{*} \omega\left(\sum_{j} y_{j} \frac{\partial}{\partial y_{j}}, \gamma_{*}^{-1} \cdot\right)\right) \\
& =\left(\gamma \cdot x, \gamma^{-1, *} \sum_{j} y_{j} d x_{j}\right) .
\end{aligned}
$$

Thus

$$
\begin{aligned}
\gamma \cdot L\left(y, \sigma_{k}(y)\right) & =\left\{\left(\gamma \cdot x, \gamma \cdot\left(y_{1}+\sigma_{k, 1}(y)(x), \cdots, y_{n}+\sigma_{k, n}(y)(x)\right)\right) \mid x \in T^{n}\right\} \\
& =\left\{\left(\gamma \cdot x, \gamma^{-1, *} \sum_{j}\left(y_{j}+\sigma_{k, j}(y)(x)\right) d x_{j} \mid x \in T^{n}\right\}\right. \\
& =L\left(\gamma \cdot y, \gamma^{-1, *} \sigma_{k}(y)\right),
\end{aligned}
$$

for any $\gamma \in \Gamma$ and $y \in B_{h_{E}}(0,2 r)$. Since the $\Gamma$-action preserves $\omega_{k}$ and $\Omega_{k}$, $L\left(\gamma \cdot y, \gamma^{-1, *} \sigma_{k}(y)\right)$ are special Lagrangian submanifolds. By the uniqueness of $\sigma_{k}(y), \gamma^{-1, *} \sigma_{k}(y)=\sigma_{k}(\gamma \cdot y) \in C^{1, \alpha}\left(d \Omega^{0}\left(T^{n}\right) \oplus d^{* h} \Omega^{2}\left(T^{n}\right)\right)$. Hence

$$
\begin{aligned}
\Psi_{k}(\gamma \cdot x, \gamma \cdot y) & =\left(\gamma \cdot x, \gamma \cdot y+\sigma_{k}(\gamma \cdot y)\right) \\
& =\left(\gamma \cdot x, \gamma^{-1, *} \sum_{j}\left(y_{j}+\sigma_{k, j}(y)\right) d x_{j}\right) \\
& =\left(\gamma \cdot x, \gamma \cdot\left(y_{1}+\sigma_{k, 1}(y), \cdots, y_{n}+\sigma_{k, n}(y)\right)\right) \\
& =\gamma \cdot \Psi_{k}(x, y),
\end{aligned}
$$

i.e. $\Psi_{k}$ is a $\Gamma$-equivariant map.

We denote by $\mathcal{P}: Y_{2 r} \longrightarrow B_{h_{E}}(0,2 r)$ the natural projection, $B_{k}=B_{h_{E}}\left(0, \frac{3}{2} r\right)$ and $W_{k}=\Psi_{k}\left(Y_{\frac{3}{2} r}\right)$. Since the $\Gamma$-action on $B_{h_{E}}(0,2 r)$ preserves the metric $h_{E}$ and $0, B_{k}=B_{h_{E}}\left(0, \frac{3}{2} r\right)$ is invariant. By $\delta \ll 1 \ll r, W_{k} \supset Y_{r}$. Then $f_{k}=\mathcal{P} \circ \Psi_{k}^{-1}: W_{k} \longrightarrow B_{k}$ is a $\Gamma$-equivariant special Lagrangian fibration of $\left(W_{k}, \omega_{k}, \Omega_{k}\right)$ of phase $\theta_{k}$. We obtain the conclusion. 
5. Proof of Theorem 1.1. Now we are ready to prove Theorem 1.1 .

Proof of Theorem 1.1. Assume that the conclusion is not true. Then, for any fixed $\sigma>1$, there is a family of closed Ricci-flat Calabi-Yau $n$-manifolds $\left\{\left(M_{k}, \omega_{k}, J_{k}, g_{k}, \Omega_{k}\right)\right\}$ with $\left[\omega_{k}\right] \in H^{2}\left(M_{k}, \mathbb{Z}\right)$, and $p_{k} \in M_{k}$ such that

i) the injectivity radius and the sectional curvature fulfil the following estimates:

$$
i_{g_{k}}\left(p_{k}\right)<\frac{1}{k}, \quad \sup _{B_{g_{k}}\left(p_{k}, 1\right)}\left|K_{g_{k}}\right| \leq 1,
$$

ii) $\left[\left.\Omega_{k}\right|_{B_{g_{k}}\left(p_{k}, \sigma i_{g_{k}}\left(p_{k}\right)\right)}\right] \neq 0$ in $H^{n}\left(B_{g_{k}}\left(p_{k}, \sigma i_{g_{k}}\left(p_{k}\right)\right), \mathbb{C}\right)$,

iii) for any open subset $W_{k}^{\prime} \supset B_{g_{k}}\left(p_{k}, \sigma i_{g_{k}}\left(p_{k}\right)\right),\left(W_{k}^{\prime}, \omega_{k}, \Omega_{k}\right)$ would not admit special Lagrangian fibrations.

If we denote $\tilde{\omega}_{k}=i_{g_{k}}^{-2}\left(p_{k}\right) \omega_{k}, \tilde{g}_{k}=i_{g_{k}}^{-2}\left(p_{k}\right) g_{k}$, and $\tilde{\Omega}_{k}=i_{g_{k}}^{-n}\left(p_{k}\right) \Omega_{k}$, then

$$
i_{\tilde{g}_{k}}\left(p_{k}\right)=1, \quad \sup _{B_{\tilde{g}_{k}}\left(p_{k}, k\right)}\left|K_{\tilde{g}_{k}}\right| \leq \frac{1}{k^{2}},
$$

and $\left[\left.\tilde{\Omega}_{k}\right|_{B_{\tilde{g}_{k}}\left(p_{k}, \sigma\right)}\right] \neq 0$ in $H^{n}\left(B_{\tilde{g}_{k}}\left(p_{k}, \sigma\right), \mathbb{C}\right)$. By the Cheeger-Gromov convergence theorem (cf. Theorem 2.4), a subsequence of $\left(M_{k}, \tilde{\omega}_{k}, \tilde{g}_{k}, J_{k}, \tilde{\Omega}_{k}, p_{k}\right)$ converges to a complete flat Calabi-Yau $n$-manifold $\left(X, \omega_{0}, g_{0}, J_{0}, \Omega_{0}, p_{0}\right)$ in the $C^{\infty}$-sense, i.e. for any $r>\sigma$, there are embeddings $F_{r, k}: B_{g_{0}}\left(p_{0}, r\right) \longrightarrow M_{k}$ such that $F_{r, k}\left(p_{0}\right)=p_{k}$, and $F_{r, k}^{*} \tilde{g}_{k}$ (resp. $F_{r, k}^{*} \tilde{\omega}_{k}$ and $F_{r, k}^{*} \tilde{\Omega}_{k}$ ) converges to $g_{0}$ (resp. $\omega_{0}$ and $\left.\Omega_{0}\right)$ in the $C^{\infty}$-sense. Furthermore, $i_{g_{0}}\left(p_{0}\right)=1$. The soul theorem (cf. [10], [34] implies that there is a compact flat totally geodesic submanifold $S \subset X$, the soul, such that $\left(X, g_{0}\right)$ is isometric to the total space of the normal bundle $\nu(S)$ with a metric induced by $\left.g_{0}\right|_{S}$ and a natural flat connection.

By Proposition (3.4), there is a finite normal covering $\pi: \tilde{X} \longrightarrow X$ with covering group $\Gamma$ such that

i) $\left(\tilde{X}, \pi^{*} g_{0}\right)$ is isometric to $\left(T^{n} \times \mathbb{R}^{n}, h+h_{E}\right)$, where $T^{n}=\mathbb{R}^{n} / \Lambda, \Lambda$ is a lattice in $\mathbb{R}^{n}, h_{E}$ is the standard Euclidean metric on $\mathbb{R}^{n}$, and $h$ is the standard flat metric on $T^{n}$ induced by $h_{E}$.

ii) The action of $\Gamma$ on $\tilde{X}$ is a product action, i.e. there are $\Gamma$-actions on $T^{n}$ and $\mathbb{R}^{n}$ such that $\gamma \cdot(x, y)=(\gamma \cdot x, \gamma \cdot y)$ for any $x \in T^{n}$ and $y \in \mathbb{R}^{n}$. Furthermore, $T^{n} \times\{0\}$ is $\Gamma$-invariant, and $S=\left(T^{n} \times\{0\}\right) / \Gamma$.

iii)

$$
\left.\pi^{*} \omega_{0}\right|_{T^{n} \times\{y\}} \equiv 0, \text { and }\left.\pi^{*} \operatorname{Im} e^{\sqrt{-1} \theta_{0}} \Omega_{0}\right|_{T^{n} \times\{y\}} \equiv 0,
$$

for any $y \in \mathbb{R}^{n}$, and a constant $\theta_{0} \in \mathbb{R}$.

Note that the $\Gamma$-action on $\mathbb{R}^{n}$ preserves $h_{E}$ and $B_{h_{E}}(0, \rho)$, which implies that $\tilde{X}_{\rho}=T^{n} \times B_{h_{E}}(0, \rho)$ is invariant, for any $\rho>0$. Lemma 3.3 shows $\left[\left.F_{r, k}^{*} \tilde{\omega}_{k}\right|_{S}\right]=0$ in $H^{2}(S, \mathbb{R})$, for $k \gg 1$, which implies $\left[\left.\pi^{*} F_{r, k}^{*} \tilde{\omega}_{k}\right|_{\tilde{X}_{\rho}}\right]=0$ 
in $H^{2}\left(\tilde{X}_{\rho}, \mathbb{R}\right)$, for any $\rho>0$. By Remark 3.5 , there are parallel 1-forms $d x_{1}, \cdots, d x_{n}$ on $\left(T^{n}, h\right)$, which are pointwise linear independent, and coordinates $y_{1}, \cdots, y_{n}$ on $\mathbb{R}^{n}$ such that

$\pi^{*} g_{0}=\sum\left(d x_{j}^{2}+d y_{j}^{2}\right), \quad \pi^{*} \omega_{0}=\sum d x_{j} \wedge d y_{j}, \quad e^{\sqrt{-1} \theta_{0}} \pi^{*} \Omega_{0}=\bigwedge_{j=1}^{n}\left(d x_{j}+\sqrt{-1} d y_{j}\right)$.

Hence Condition 4.1 is satisfied.

Let $r>\rho \gg \sigma$ be such that $B_{g_{0}}\left(p_{0}, \sigma\right) \subset \pi\left(\tilde{X}_{\rho}\right) \subset \pi\left(\tilde{X}_{2 \rho}\right) \subset B_{g_{0}}\left(p_{0}, r\right)$. By Proposition (4.6), for $k \gg 1$, there is an open set $W_{k} \supset \tilde{X}_{\rho}$ such that $\left(W_{k}, \pi^{*} F_{r, k}^{*} \omega_{k}, \pi^{*} \bar{F}_{r, k}^{*} \Omega_{k}\right)$ admits an equivariant special Lagrangian fibration $f_{k}: W_{k} \longrightarrow B_{k}$ of phase $\theta_{k}$, where $B_{k} \subset \mathbb{R}^{n}$, i.e. there is a $\Gamma$-action on $B_{k}, f_{k}$ is a $\Gamma$-equivariant map, and

$$
\left.\pi^{*} F_{r, k}^{*} \omega_{k}\right|_{f_{k}^{-1}(b)} \equiv 0,\left.\quad \pi^{*} F_{r, k}^{*} \operatorname{Im} e^{\sqrt{-1} \theta_{k}} \Omega_{k}\right|_{f_{k}^{-1}(b)} \equiv 0,
$$

for any $b \in B_{k}$. Hence $f_{k}$ induces a special Lagrangian fibration $\bar{f}_{k}: \pi\left(W_{k}\right) \longrightarrow$ $B_{k} / \Gamma$, which implies that $\left(F_{r, k} \circ \pi\left(W_{k}\right), \omega_{k}, \Omega_{k}\right)$ admits a special Lagrangian fibration, and $F_{r, k} \circ \pi\left(W_{k}\right) \supset B_{g_{k}}\left(x_{k}, \sigma i_{g_{k}}\left(x_{k}\right)\right)$. It is a contradiction. We obtain the conclusion.

6. Estimates for injectivity radius. In this section, we prove Theorem 1.5 (Corollary 6.1) and Theorem 1.6 (Theorem 6.3). The following estimate for injectivity radius is a direct consequence of Theorem 2.5 .

Corollary 6.1. Let $(M, g)$ be a closed Riemannian manifold, $\Theta$ be a calibration $n$-form, and $p \in M$. Assume that the sectional curvature $K_{g}$ satisfies

$$
\sup _{B_{g}(p, 2 \pi)} K_{g} \leq 1
$$

and there is a submanifold $L$ calibrated by $\Theta$ such that $\operatorname{dim}_{\mathbb{R}} L=n, p \in L$, and

$$
\int_{L} \Theta<\frac{\pi}{2 n} \varpi_{n-1}
$$

where $\varpi_{n-1}$ is the volume of $S^{n-1}$ with the standard metric of constant curvature 1. Then the injectivity radius $i_{g}(p)$ of $(M, g)$ at $p$ satisfies

$$
i_{g}(p)^{n} \leq \frac{n \pi^{n-1}}{2^{n-1} \varpi_{n-1}} \int_{L} \Theta
$$

Proof of Corollary6.1 and Theorem 1.5. By Theorem 2.5, we have

$$
\operatorname{Vol}_{h_{1}}\left(B_{h_{1}}(r)\right) \leq \operatorname{Vol}_{g}\left(B_{g}(p, r) \cap L\right) \leq \operatorname{Vol}_{g}(L)=\int_{L} \Theta,
$$

for any $r \leq \min \left\{i_{g}(p), \frac{\pi}{2}\right\}$, where $h_{1}$ denotes the standard metric on $S^{n}$ with constant curvature 1 , and $B_{h_{1}}(r)$ denotes a metric $r$-ball in $S^{n}$. Since $h_{1}=$ 
$d r^{2}+\sin ^{2} r h_{S^{n-1}}$ where $h_{S^{n-1}}$ is the standard metric on $S^{n-1}$ with constant curvature 1 , we obtain $\sin r \geq \frac{2}{\pi} r$, and

$$
\frac{2^{n-1}}{n \pi^{n-1}} r^{n} \varpi_{n-1} \leq \int_{0}^{r} \sin ^{n-1} r d r \varpi_{n-1}=\operatorname{Vol}_{h_{1}}\left(B_{h_{1}}(r)\right) \leq \int_{L} \Theta .
$$

If $i_{g}(p) \geq \frac{\pi}{2}$, by letting $r=\frac{\pi}{2}$, we obtain

$$
\frac{\pi}{2 n} \varpi_{n-1} \leq \int_{L} \Theta<\frac{\pi}{2 n} \varpi_{n-1},
$$

which is a contradiction. Thus $i_{g}(p)<\frac{\pi}{2}$. By letting $r=i_{g}(p)$, we obtain

$$
i_{g}(p)^{n} \leq \frac{n \pi^{n-1}}{2^{n-1} \varpi_{n-1}} \int_{L} \Theta .
$$

We obtain Theorem 1.5 by applying the above arguments to special Lagrangian submanifolds in Ricci-flat Calabi-Yau manifolds.

An obvious application of Corollary 6.1 is estimating injectivity radii by volumes of holomorphic submanifolds, which is interesting by itself.

Corollary 6.2. Let $(M, \omega, J, g)$ be a closed Kähler $m$-manifold, and $p \in$ $M$. Assume that the sectional curvature $K_{g}$ satisfies

$$
\sup _{M} K_{g} \leq 1
$$

and there is a smooth holomorphic n-submanifold $N$ such that $p \in N$, and

$$
\int_{N} \omega^{n}<\frac{(n-1) ! \pi}{2} \varpi_{n-1} .
$$

Then the injectivity radius $i_{g}(p)$ of $(M, g)$ at $p$ satisfies

$$
i_{g}(p)^{n} \leq \frac{\pi^{n-1}}{(n-1) ! 2^{n-1} \varpi_{n-1}} \int_{N} \omega^{n} .
$$

By combining this corollary and the result in $[\mathbf{1 0}$, there are $F$-structures of positive rank on the regions of Kähler manifolds with bounded curvature and fibred by holomorphic submanifolds with small volumes.

Finally, we estimate the volume of metric balls when they are fibred by calibrated submanifolds with small volume in the absence of bounded curvature of the ambient manifolds.

Theorem 6.3. For any $n, m \in \mathbb{N}$ and any $\varepsilon>0$, there is a constant $\delta=\delta(n, m, \varepsilon)>0$ for which the following statement holds true: Assume that $(M, g, \Theta)$ is a closed Ricci-flat Einstein m-manifold with a calibration $n$-form $\Theta, p \in M$, and there is a homology class $A \in H_{n}(M, \mathbb{Z})$ such that for any $x \in$ 
$B_{g}(p, 1)$, there is a n-submanifold $L_{x}$ calibrated by $\Theta$ passing $x$ and presenting A, i.e. $x \in L_{x}$ and $\left[L_{x}\right]=A$. If

$$
\int_{A} \Theta<\delta
$$

then

$$
\operatorname{Vol}_{g}\left(B_{g}(p, 1)\right) \leq \varepsilon .
$$

Proof of Theorem 6.3 and Theorem 1.6. Assume that the conclusion is not true. Then there is a family of closed Ricci-flat Einstein $m$-manifolds $\left\{\left(M_{k}, g_{k}, \Theta_{k}\right)\right\}$ with calibration $n$-forms $\Theta_{k}, p_{k} \in M_{k}$, and there are homology class $A_{k} \in H_{n}\left(M_{k}, \mathbb{Z}\right)$ such that

i) for any $x \in B_{g_{k}}\left(p_{k}, 1\right)$, there is a $n$-submanifold $L_{k, x}$ calibrated by $\Theta_{k}$ passing $x$ and presenting $A_{k}$, i.e. $x \in L_{k, x}$ and $\left[L_{k, x}\right]=A_{k}$,

ii)

$$
\int_{A_{k}} \Theta_{k}<\frac{1}{k}
$$

However,

$$
\operatorname{Vol}_{g_{k}}\left(B_{g_{k}}\left(p_{k}, 1\right)\right) \geq C,
$$

for a constant $C>0$ independent of $k$.

By the Gromov pre-compactness theorem (Theorem 2.2), by passing to a subsequence, $\left\{\left(M_{k}, g_{k}, p_{k}\right)\right\}$ converges to a complete path metric space $\left(Y, d_{Y}, p\right)$ in the pointed Gromov-Hausdorff sense. By Theorem 2.3, the Hausdorff dimension of $\left(Y, d_{Y}, p\right)$ is $m$, and there is a closed subset $S_{Y} \subset Y$ of Hausdorff dimension smaller than $m-1$, i.e. $\operatorname{dim}_{\mathcal{H}} S_{Y}<m-1$, such that $Y \backslash S_{Y}$ is a smooth manifold, and $d_{Y}$ is induced by a Ricci-flat Einstein metric $g_{\infty}$ on $Y \backslash S_{Y}$. Furthermore, for any compact subset $D \subset Y \backslash S_{Y}$, there are embeddings $F_{k, D}: D \longrightarrow M_{k}$ such that $F_{k, D}^{*} g_{k}$ converges to $g_{\infty}$ in the $C^{\infty}$-sense.

We take $D$ so large that $D \cap B_{d_{Y}}\left(p, \frac{1}{8}\right)$ is not empty. For a $y \in \operatorname{int} D \cap$ $B_{d_{Y}}\left(p, \frac{1}{4}\right)$, there is a $r>0$ such that $B_{g_{\infty}}(y, 2 r) \subset \operatorname{int} D \cap B_{d_{Y}}\left(p, \frac{1}{4}\right)$, and $B_{g_{k}}\left(F_{k, D}(y), r\right) \subset F_{k, D}(D) \cap B_{g_{k}}\left(p_{k}, \frac{1}{2}\right)$ when $k \gg 1$. By the smooth convergence of $F_{k, D}^{*} g_{k}$, the sectional curvatures $K_{g_{k}}$ satisfy

$$
\sup _{F_{k, D}(D)}\left|K_{g_{k}}\right| \leq C_{D}
$$

for a constant $C_{D}$ depending on $D$, but independent of $k$. Let $L_{k}$ be an $n$ submanifold calibrated by $\Theta_{k}$ passing $F_{k, D}(y)$ and presenting $A_{k}$, i.e. $F_{k, D}(y) \in$ $L_{k}$ and $\left[L_{k}\right]=A_{k}$. By the Bishop-Gromov comparison theorem, for any $\rho \leq r$,

$$
\begin{gathered}
\frac{\operatorname{Vol}_{g_{k}}\left(B_{g_{k}}\left(F_{k, D}(y), \rho\right)\right)}{\rho^{m}} \geq \operatorname{Vol}_{g_{k}}\left(B_{g_{k}}\left(F_{k, D}(y), 1\right)\right) \geq \operatorname{Vol}_{g_{k}}\left(B_{g_{k}}\left(p_{k}, \frac{1}{2}\right)\right) \\
\geq \frac{1}{2^{m}} \operatorname{Vol}_{g_{k}}\left(B_{g_{k}}\left(p_{k}, 1\right)\right) \geq \frac{C}{2^{m}} .
\end{gathered}
$$


Then there is a uniform lower bound $\iota>0$ for injectivity radii $i_{g_{k}}\left(F_{k, D}(y)\right)$ at $F_{k, D}(y)$ (cf. 34), i.e. $i_{g_{k}}\left(F_{k, D}(y)\right) \geq \iota>0$ for $k \gg 1$. By Theorem 2.5, we have

$$
\operatorname{Vol}_{h_{1}}\left(B_{h_{1}}(\rho)\right) \leq \operatorname{Vol}_{g_{k}}\left(B_{g_{k}}\left(F_{k, D}(y), \rho\right) \cap L_{k}\right) \leq \int_{L_{k}} \Theta_{k}=\int_{A_{k}} \Theta_{k} \leq \frac{1}{k}
$$

where $\rho=\min \left\{\iota, r, \frac{\pi}{\sqrt{C_{D}}}\right\}, h_{1}$ denotes the standard metric on $S^{n}$ with constant curvature $C_{D}$, and $B_{h_{1}}(\rho)$ denotes a metric $\rho$-ball in $S^{n}$. We obtain a contradiction when $k \gg 1$, and, thus, we obtain the conclusion.

We obtain Theorem 1.6 by applying the above arguments to special Lagrangian submanifolds in Ricci-flat Calabi-Yau manifolds.

REMark 6.4. Let $\left\{\left(M_{k}, g_{k}, \Theta_{k}\right)\right\}$ be a family of closed Ricci-flat Einstein $m$-manifolds with calibration $n$-forms $\Theta_{k}$, and $\left\{p_{k}\right\}$ be a sequence of points such that there are open subsets $W_{k} \supset B_{g_{k}}\left(p_{k}, 1\right)$ in $M_{k}$ admitting calibrated fibrations $f_{k}: W_{k} \longrightarrow B_{k}$ corresponding to $\Theta_{k}$, i.e. for any $b_{k} \in B_{k}, f_{k}^{-1}\left(b_{k}\right)$ is an $n$-submanifold calibrated by $\Theta_{k}$. If

$$
\lim _{k \longrightarrow \infty} \int_{f_{k}^{-1}\left(b_{k}\right)} \Theta_{k}=0
$$

where $b_{k} \in B_{k}$, Theorem 6.3 implies that

$$
\lim _{k \longrightarrow \infty} \operatorname{Vol}_{g_{k}}\left(B_{g_{k}}\left(p_{k}, 1\right)\right)=0,
$$

and, by passing to a subsequence, $\left\{\left(M_{k}, g_{k}\right)\right\}$ converges to a path metric space of a lower Hausdorff dimension in the pointed Gromov-Hausdorff sense.

REMARK 6.5. Theorem 6.3 applies to $G_{2}$-manifolds and $\operatorname{Spin}(7)$-manifolds since they are Ricci-flat Einstein manifolds (cf. [28]).

\section{References}

1. Anderson M.T., Ricci curvature bounds and Einstein metrics on compact manifolds, J. Amer. Math. Soc., 2 (1989), 455-490.

2. Anderson M.T., Convergence and rigidity of manifolds under Ricci curvature bounds, Invent. Math., 102 (1990), 429-445.

3. Anderson M.T., The $L^{2}$ structure of moduli spaces of Einstein metrics on 4-manifolds, G.A.F.A., (1991), 231-251.

4. Cheeger J., Degeneration of Einstein metrics and metrics with special holonomy, in Surveys in differential geometry VIII, 29-73.

5. Cheeger J., Colding T. H., On the structure of space with Ricci curvature bounded below I, Jour. of Diff. Geom., 46 (1997), 406-480.

6. Cheeger J., Colding T. H., On the structure of space with Ricci curvature bounded below II, Jour. of Diff. Geom., 52 (1999), 13-35.

7. Cheeger J., Colding T.H., Tian G., On the singularities of spaces with bounded Ricci curvature, Geom. Funct. Anal., 12 (2002), 873-914. 
8. Cheeger J., Fukaya K., Gromov M., Nilpotent structures and invariant metrics on collapsed manifolds, Joural of the American Mathematical Society, 5 (1992), 327-372.

9. Cheeger J., Gromov M., Collapsing Riemannian manifolds while keeping their curvature bound I, J. Differ. Geom., 23 (1986), 309-364.

10. Cheeger J., Gromov M., Collapsing Riemannian manifolds while keeping their curvature bounded II, J. Diff. Geom., 32 (1990), 269-298.

11. Cheeger J., Tian G., Anti-self-duality of curvature and degeneration of metrics with special holonomy, Commun. Math. Phys., 255 (2005), 391-417.

12. Cheeger J., Tian G., Curvature and injectivity radius estimates for Einstein 4-manifolds, Journal of the American Mathematical Society, 19 (2006), 487-525.

13. Duistermaat J., On global action-angle coordinates, Comm. Pure Appled Math., 33 (1980), 687-706.

14. Fukaya K., Hausdorff convergence of Riemannian manifolds and its application, Advance Studies in Pure Mathematics, 18 (1990), 143-234.

15. Fukaya K., Multivalued Morse theory, asymptortic analysis and mirror aymmetry, Proceedings of Symposia in Pure Mathematics, 73 (2005), 205-278.

16. Green R. E., Wu H., Lipschitz converges of Riemannian manifolds, Pacific J. Math., 131 (1988), 119-141.

17. Gromov M., Metric structures for Riemannian and non-Riemannian spaces, Birkhäuser 1999.

18. Goldstein E., A construction of new families of minimal lagrangian submanifolds via torus action, J. Diff. Geom., 58 (2001), 233-261.

19. Goldstein E., Calibrated fibrations, Comm. Anal. Geom., 10 (2002), 127-150.

20. Gross M., Examples of special lagrangian fibrations, in Symplectic Geometry and Mirror Symmetry, World Scientific Singapore, (2001), 81-109.

21. Gross M., Special lagrangian fibrations II-Geometry, Surveys in Differential Geometry: Differential geometry inspired by string theory, International Press, (1999), 341-404.

22. Gross M., Wilson P. M. H., Mirror symmetry via 3-tori for a class of Calabi-Yau treefolds, Math. Ann., 309 (1997), 505-531.

23. Gross M., Wilson P. M. H., Large complex structure limits of K3 surfaces, J. Diff. Geom., 55 (2000), 475-546.

24. Gilbarg D., Trudinger N. S., Elliptic partial differential equations of second two, Springer 1983.

25. Gukov S., Yau S. T., Zaslow E., Duality and fibrations on $G_{2}$ manifolds, Turkish Journal of Mathematics, 27 (2003), 61-97.

26. Harvey R., Lawson H. B., Calibrated geometries, Acta Math., 148 (1982), 47-157.

27. Hitchin N., The moduli space of special lagrangian submanifolds, Ann. Scuola Norm. Sup. Pisa Cl. Sci., 25 (1997), 503-515.

28. Joyce D. D., Compact manifolds with special holonomy, Oxford University Press, 2000.

29. Joyce D. D., Singularities of special lagrangian fibrations and the $S Y Z$ conjecture, Comm. Anal. Geom., 11 (2003), 859-907.

30. Joyce D. D., Lectures on Calabi-Yau and special Lagrangian geometry, math.DG/0108088.

31. Kontsevich M., Soibelman Y., Homological mirror symmetry and torus fibrations, in Symplectic geometry and mirror symmetry, World Sci. Publishing, (2001), 203-263.

32. Lu P., Kähler-Einstein metrics on Kummer threefold and special lagrangian tori, Comm. Anal. Geom., 7 (1999), 787-806.

33. Mclean R. C., Deformation of calibrated submanifolds, Comm. Anal. Geom., 6 (1998), 705-747. 
34. Petersen P., Riemannian Geometry, Springer, 1997.

35. Ruan W. D., On the convergence and collapsing of Kähler metrics, J. Differ. Geom., 52 (1999), 1-40.

36. Ruan W. D., Generalized special Lagrangian torus fibration for Calabi-Yau hypersurfaces in toric varieties. I. Commun. Contemp. Math., 9 (2007), no. 2, 201-216.

37. Ruan W. D., Generalized special Lagrangian torus fibrations for Calabi-Yau hypersurfaces in toric varieties. II. in Mirror symmetry. V, Amer. Math. Soc., Providence, RI, 2006. 457-477.

38. Ruan W.D., Generalized special Lagrangian fibration for Calabi-Yau hypersurfaces in toric varieties III: The smooth fibres, arXiv:math/0309450.

39. Ruan W. D., Zhang Y., Convergence of Calabi-Yau manifolds, Advances in Mathematics Volume 228, Issue 3, (2011), 1543-1589.

40. Salur S., Deformations of special lagrangian submanifolds, Comm. Cont. Math., Vol. 2, 3 (2000), 365-372.

41. Strominger A., Yau S. T., Zaslow E., Mirror symmetry is T-duality, Nucl. Phys. B, 479 (1996), 243-259.

42. Thomas R.P., Yau S.T., Special Lagrangians, stable bundles and mean curvature flow, Comm. Anal. Geom., 10 (2002), no. 5, 1075-1113.

43. Tosatti V., Limits of Calabi-Yau metrics when the Kähler class degenerates, J. Eur. Math. Soc., 11 (2009), no. 4, (2009), 755-776.

44. Yau S. T., On the Ricci curvature of a compact Kähler manifold and complex MongeAmpere equation I, Comm. Pure Appl. Math., 31 (1978), 339-411.

45. Yau S. T., Einstein manifolds with zero Ricci curvature, in Lectures on Einstein manifolds, International Press, (1999), 1-14.

Received August 06, 2016

Tsinghua University

Yau Mathematical Sciences Center

Beijing 100084

P. R. China

e-mail: yuguangzhang76@yahoo.com 